\title{
Convex Relaxation for Optimal Power Flow Problem: Mesh Networks
}

\author{
Ramtin Madani, Somayeh Sojoudi and Javad Lavaei
}

\begin{abstract}
This paper is concerned with the optimal power flow (OPF) problem. We have recently shown that a convex relaxation based on semidefinite programming (SDP) is able to find a global solution of OPF for IEEE benchmark systems, and moreover this technique is guaranteed to work over acyclic (distribution) networks. The present work studies the potential of the SDP relaxation for OPF over mesh (transmission) networks. First, we consider a simple class of cyclic systems, namely weakly-cyclic networks with cycles of size 3 . We show that the success of the SDP relaxation depends on how the line capacities are modeled mathematically. More precisely, the SDP relaxation is proven to succeed if the capacity of each line is modeled in terms of bus voltage difference, as opposed to line active power, apparent power or angle difference. This result elucidates the role of the problem formulation. Our second contribution is to relate the rank of the minimum-rank solution of the SDP relaxation to the network topology. The goal is to understand how the computational complexity of OPF is related to the underlying topology of the power network. To this end, an upper bound is derived on the rank of the SDP solution, which is expected to be small in practice. A penalization method is then applied to the SDP relaxation to enforce the rank of its solution to become 1 , leading to a near-optimal solution for OPF with a guaranteed optimality degree. The remarkable performance of this technique is demonstrated on IEEE systems with more than 7000 different cost functions.
\end{abstract}

\section{INTRODUCTION}

The optimal power flow (OPF) problem aims to find an optimal operating point of a power system, which minimizes a certain objective function (e.g., power loss or generation cost) subject to network and physical constraints [1]. Due to the nonlinear interrelation among active power, reactive power and voltage magnitude, OPF is described by nonlinear equations and may have a nonconvex/disconnected feasibility region. Since 1962, the nonlinearity of the OPF problem has been studied, and various heuristic and local-search algorithms have been proposed [2], [3].

The paper [4] proposes two methods for solving OPF: (i) to use a convex relaxation based on semidefinite programming (SDP), (ii) to solve the SDP-type Lagrangian dual of OPF. That work shows that the SDP relaxation is exact if and only if the duality gap is zero. More importantly, [4] makes the observation that OPF has a zero duality gap for IEEE benchmark systems with 14, 30, 57, 118 and 300 buses, in addition to several randomly generated power networks. This technique is the first method proposed since the introduction of the OPF problem that is able to find a provably global solution for practical OPF problems. The SDP relaxation for OPF has

Ramtin Madani and Javad Lavaei are with the Electrical Engineering Department, Columbia University (emails: madani@ee.columbia.edu and lavaei@ee.columbia.edu). Somayeh Sojoudi is with the Langone Medical Center, New York University (email: somayeh.sojoudi@nyumc.org). This work was supported by the NSF CAREER Award 1351279 and a Google Faculty Award. attracted much attention due to its ability to find a global solution in polynomial time, and it has been applied to various applications in power systems including: voltage regulation in distribution systems [5], state estimation [6], calculation of voltage stability margin [7], economic dispatch in unbalanced distribution networks [8], charging of electric vehicles [9], and power management under time-varying conditions [10].

The paper [11] shows that the SDP relaxation is exact in two cases: (i) for acyclic networks, (ii) for cyclic networks after relaxing the angle constraints (similar result was derived in [12] and [13] for acyclic networks). This exactness was related to the passivity of transmission lines and transformers. A question arises as to whether the SDP relaxation remains exact for mesh (cyclic) networks without any angle relaxations. To address this problem, the paper [14] shows that the relaxation is not always exact for a three-bus cyclic network. More examples can be found in the recent paper [15], where the existence of local solutions is studied for the OPF problem. To improve the performance of the above-mentioned convex relaxation, the papers [16] and [17] suggest solving a sequence of SDPtype relaxations based on the branch and bound technique. However, it is highly desirable to develop an algorithm needing to solve only a few SDP relaxations in order to guarantee a polynomial-time run for the algorithm. The aim of this paper is to investigate the possibility of finding a global or near-global solution of the OPF problem for mesh networks by solving only a few SDP relaxations.

In this work, we first consider the three-bus system studied in [14] and prove that the exactness of the SDP relaxation depends on the problem formulation. More precisely, we show that there are four (almost) equivalent ways to model the capacity of a power line but only one of these models always gives rise to the exactness of the SDP relaxation. We also prove that the relaxation remains exact for weakly-cyclic networks with cycles of size 3 . Furthermore, we substantiate that this type of network has a convex injection region in the lossless case and a non-convex injection region with a convex Pareto front in the lossy case. The importance of this result is that the SDP relaxation works on certain cyclic networks, for example the ones generated from three-bus subgraphs (this type of network is related to three-phase systems).

In the case when the SDP relaxation does not work, an upper bound is provided on the rank of the minimum-rank solution of the SDP relaxation. This bound is related only to the structure of the power network and this number is expected to be very small for real-world power networks. Finally, a heuristic method is proposed to enforce the SDP relaxation to produce a rank-1 solution for general networks (by somehow eliminating the undesirable eigenvalues of the low-rank solution). The efficacy of the proposed technique is elucidated by extensive simulations on IEEE systems as well 


\begin{tabular}{|c|c|}
\hline OPF Problem & SDP Relaxation of OPF \\
\hline $\begin{array}{l}\text { Minimize } \sum_{k \in \mathcal{G}} f_{k}\left(P_{G_{k}}\right) \text { over } \mathbf{P}_{G}, \mathbf{Q}_{G}, \mathbf{V} \\
\text { Subject to: }\end{array}$ & $\begin{array}{l}\text { Minimize } \sum_{k \in \mathcal{G}} f_{k}\left(P_{G_{k}}\right) \text { over } \mathbf{P}_{G}, \mathbf{Q}_{G}, \mathbf{W} \in \mathbb{H}_{+}^{n} \\
\text { Subject to: }\end{array}$ \\
\hline 1- A capacity constraint for each line $(l, m) \in \mathcal{L}$ & 1- A convexified capacity constraint for each line \\
\hline 2- The following constraints for each bus $k \in \mathcal{N}$ : & 2- The following constraints for each bus $k \in \mathcal{N}$ : \\
\hline$P_{G_{k}}-P_{D_{k}}=\sum_{l \in \mathcal{N}(k)} \operatorname{Re}\left\{V_{k}\left(V_{k}^{*}-V_{l}^{*}\right) y_{k l}^{*}\right\}$ & $P_{G_{k}}-P_{D_{k}}=\sum_{l \in \mathcal{N}(k)} \operatorname{Re}\left\{\left(W_{k k}-W_{k l}\right) y_{k l}^{*}\right\} \quad$ (2a) \\
\hline$Q_{G_{k}}-Q_{D_{k}}=\sum_{l \in \mathcal{N}(k)} \operatorname{Im}\left\{V_{k}\left(V_{k}^{*}-V_{l}^{*}\right) y_{k l}^{*}\right\} \quad(1 \mathrm{~b})$ & $Q_{G_{k}}-Q_{D_{k}}=\sum_{l \in \mathcal{N}(k)} \operatorname{Im}\left\{\left(W_{k k}-W_{k l}\right) y_{k l}^{*}\right\}(2 \mathrm{~b})$ \\
\hline$P_{k}^{\min } \leq P_{G_{k}} \leq P_{k}^{\max }$ & $P_{k}^{\min } \leq P_{G_{k}} \leq P_{k}^{\max }$ \\
\hline$Q_{k}^{\min } \leq Q_{G_{k}} \leq Q_{k}^{\max }$ & $Q_{k}^{\min } \leq Q_{G_{k}} \leq Q_{k}^{\max }$ \\
\hline$V_{k}^{\min } \leq\left|V_{k}\right| \leq V_{k}^{\max }$ & $\left(V_{k}^{\min }\right)^{2} \leq W_{k k} \leq\left(V_{k}^{\max }\right)^{2}$ \\
\hline
\end{tabular}

\begin{tabular}{|c|c|c|c|}
\hline \multicolumn{2}{|l|}{ Capacity constraint for line $(l, m) \in \mathcal{L}$} & \multicolumn{2}{|c|}{ Convexified capacity constraint for line $(l, m) \in \mathcal{L}$} \\
\hline$\left|\theta_{l m}\right|=\left|\measuredangle V_{l}-\measuredangle V_{m}\right| \leq \theta_{l m}^{\max }$ & (3a) & $\operatorname{Im}\left\{W_{l m}\right\} \leq \operatorname{Re}\left\{W_{l m}\right\} \tan \left(\theta_{l m}^{\max }\right)$ & (4a) \\
\hline$\left|P_{l m}\right|=\left|\operatorname{Re}\left\{V_{l}\left(V_{l}^{*}-V_{m}^{*}\right) y_{l m}^{*}\right\}\right| \leq P_{l m}^{\max }$ & $(3 b)$ & $\operatorname{Re}\left\{\left(W_{l l}-W_{l m}\right) y_{l m}^{*}\right\} \leq P_{l m}^{\max }$ & $(4 b)$ \\
\hline$\left|S_{l m}\right|=\left|V_{l}\left(V_{l}^{*}-V_{m}^{*}\right) y_{l m}^{*}\right| \leq S_{l m}^{\max }$ & $(3 \mathrm{c})$ & $\left|\left(W_{l l}-W_{l m}\right) y_{l m}^{*}\right| \leq S_{l m}^{\max }$ & $(4 c)$ \\
\hline$\left|V_{l}-V_{m}\right| \leq \Delta V_{l m}^{\max }$ & $(3 \mathrm{~d})$ & $W_{l l}+W_{m m}-W_{l m}-W_{m l} \leq\left(\Delta V_{l m}^{\max }\right)^{2}$ & $(4 d)$ \\
\hline
\end{tabular}

as a difficult example proposed in [15] for which the OPF problem has at least three local solutions. Note that this paper is concentrated on a basic OPF problem, but the results can be readily extended to a more sophisticated formulation of OPF with security constraints together with variable tap-changing transformers and capacitor banks. This can be carried out using the methodology delineated in [18].

Notations: $\mathbb{R}, \mathbb{R}_{+}, \mathbb{S}_{+}^{n}$ and $\mathbb{H}_{+}^{n}$ denote the sets of real numbers, positive real numbers, $n \times n$ positive semidefinite symmetric matrices, and $n \times n$ positive semidefinite Hermitian matrices, respectively. $\operatorname{Re}\{\mathbf{W}\}, \operatorname{Im}\{\mathbf{W}\}, \operatorname{rank}\{\mathbf{W}\}$ and trace $\{\mathbf{W}\}$ denote the real part, imaginary part, rank and trace of a given scalar/matrix $\mathbf{W}$, respectively. The notation $\mathbf{W} \succeq 0$ means that $\mathbf{W}$ is Hermitian and positive semidefinite. The notation $\measuredangle x$ denotes the angle of a complex number $x$. The notation "i" is reserved for the imaginary unit. The symbol "*”" represents the conjugate transpose operator. Given a matrix $\mathbf{W}$, its $(l, m)$ entry is denoted as $W_{l m}$. The superscript (.) opt is used to show the optimal value of an optimization parameter.

Definitions: Given a simple graph $\mathcal{H}$, its vertex and edge sets are denoted by $\mathcal{V}_{\mathcal{H}}$ and $\mathcal{E}_{\mathcal{H}}$, respectively. A "forest" is a simple graph that has no cycles and a "tree" is defined as a connected forest. A graph $\mathcal{H}^{\prime}$ is said to be a subgraph of $\mathcal{H}$ if $\mathcal{V}_{\mathcal{H}}^{\prime} \subseteq \mathcal{V}_{\mathcal{H}}$ and $\mathcal{E}_{\mathcal{H}}^{\prime} \subseteq \mathcal{E}_{\mathcal{H}}$. A subgraph $\mathcal{H}^{\prime}$ of $\mathcal{H}$ is said to be an induced subgraph if, for every pair of vertices $v_{l}, v_{m} \in \mathcal{V}_{\mathcal{H}^{\prime}},\left(v_{l}, v_{m}\right) \in$ $\mathcal{E}_{\mathcal{H}^{\prime}}$ if and only if $\left(v_{l}, v_{m}\right) \in \mathcal{E}_{\mathcal{H}}$. $\mathcal{H}^{\prime}$ is said to be induced by the vertex subset $\mathcal{V}_{\mathcal{H}^{\prime}}$.

\section{OPTIMAL POWER FLOW}

Consider a power network with the set of buses $\mathcal{N}:=$ $\{1,2, \ldots, n\}$, the set of generator buses $\mathcal{G} \subseteq \mathcal{N}$, and the set of flow lines $\mathcal{L} \subseteq \mathcal{N} \times \mathcal{N}$, where:

- A known constant-power load with the complex value $P_{D_{k}}+Q_{D_{k}}$ i is connected to each bus $k \in \mathcal{N}$.

- A generator with an unknown complex output $P_{G_{k}}+$ $Q_{G_{k}} \mathrm{i}$ is connected to each bus $k \in \mathcal{G}$.

- Each line $(l, m) \in \mathcal{L}$ of the network is modeled as a passive device with an admittance $y_{l m}$ with possible resistance and reactance (the network can be modeled as a general admittance matrix).

We call the network lossless if $\operatorname{Re}\left\{y_{l m}\right\}=0$ for all $(l, m) \in \mathcal{L}$ and call it lossy otherwise. The goal is to design the unknown outputs of all generators in such a way that the load constraints are satisfied. To formulate this problem, named optimal power flow $(O P F)$, define:

- $V_{k}$ : Unknown complex voltage at bus $k \in \mathcal{N}$.

- $P_{l m}$ : Unknown active power transferred from bus $l \in \mathcal{N}$ to the rest of the network through the line $(l, m) \in \mathcal{L}$.

- $S_{l m}$ : Unknown complex power transferred from bus $l \in$ $\mathcal{N}$ to the rest of the network through the line $(l, m) \in \mathcal{L}$.

- $f_{k}\left(P_{G_{k}}\right)$ : Known increasing, convex function representing the generation cost for generator $k \in \mathcal{G}$.

Define $\mathbf{V}, \mathbf{P}_{G}, \mathbf{Q}_{G}, \mathbf{P}_{D}$ and $\mathbf{Q}_{D}$ as the vectors $\left\{V_{k}\right\}_{k \in \mathcal{N}}$, $\left\{P_{G_{k}}\right\}_{k \in \mathcal{G}},\left\{Q_{G_{k}}\right\}_{k \in \mathcal{G}},\left\{P_{D_{k}}\right\}_{k \in \mathcal{N}}$ and $\left\{Q_{D_{k}}\right\}_{k \in \mathcal{N}}$, respectively. Given the known vectors $\mathbf{P}_{D}$ and $\mathbf{Q}_{D}$, OPF minimizes 
the total generation cost $\sum_{k \in \mathcal{G}} f_{k}\left(P_{G_{k}}\right)$ over the unknown parameters $\mathbf{V}, \mathbf{P}_{G}$ and $\mathbf{Q}_{G}$ subject to the power balance equations at all buses and some network constraints. To simplify the formulation of OPF, with no loss of generality assume that $\mathcal{G}=\mathcal{N}$. The mathematical formulation of OPF is given in (1), where:

- (1a) and (1b) are the power balance equations accounting for the conservation of energy at bus $k$.

- (1c), (1d) and (1e) restrict the active power, reactive power and voltage magnitude at bus $k$, for the given limits $P_{k}^{\min }, P_{k}^{\max }, Q_{k}^{\min }, Q_{k}^{\max }, V_{k}^{\min }, V_{k}^{\max }$.

- Each line of the network is subject to a capacity constraint to be introduced later.

- $\mathcal{N}(k)$ denotes the set of all neighboring nodes of bus $k \in \mathcal{N}$.

\section{A. Convex relaxation for optimal power flow}

Regardless of the unspecified capacity constraint, the above formulation of the OPF problem is non-convex due to the nonlinear terms $\left|V_{k}\right|$ 's and $V_{k} V_{l}^{*}$ 's. Since this problem is NPhard in the worst case, the paper [4] suggests solving a convex relaxation of OPF. To this end, notice that the constraints of OPF can all be expressed as linear functions of the entries of the quadratic matrix $\mathbf{V V}^{*}$. This implies that if the matrix $\mathbf{V V}^{*}$ is replaced by a new matrix variable $\mathbf{W} \in \mathbb{H}^{n}$, then the constraints of OPF become convex in W. Since $\mathbf{W}$ plays the role of $\mathbf{V V}^{*}$, two constraints must be added to the reformulated OPF problem in order to preserve the equivalence of the two formulations: (i) $\mathbf{W} \succeq 0$, (ii) $\operatorname{rank}\{\mathbf{W}\}=1$. Observe that Constraint (ii) is the only non-convex constraint of the reformulated OPF problem. Motivated by this fact, the SDP relaxation of OPF is defined as the OPF problem reformulated in terms of $\mathbf{W}$ under the additional constraint $\mathbf{W} \succeq 0$, which is given in (2). If the SDP relaxation gives rise to a rank-1 solution $\mathbf{W}^{\text {opt }}$, then it is said that the relaxation is exact. The exactness of the SDP relaxation is a desirable property being sought, because it implies the equivalence of the convex SDP relaxation and the non-convex OPF problem.

\section{B. Four types of capacity constraints}

In this part, the line capacity constraint in the formulation of the OPF problem given in (1) will be specified. Line flows are restricted in practice to achieve various goals such as avoiding line overheating and guaranteeing the stability of the network. Notice that

i) A thermal limit can be imposed by restricting the line active power flow $P_{l m}$, the line apparent power flow $\left|S_{l m}\right|$, or the line current magnitude $\left|I_{l m}\right|$. The maximum allowable limits on these parameters can be determined by analyzing the material characteristics of the line.

ii) A stability limit may be translated into a constraint on the voltage phase difference across the line, i.e., $\left|\measuredangle V_{l}-\measuredangle V_{m}\right|$.

Hence, each line $(l, m) \in \mathcal{L}$ may be associated with one or multiple capacities constraints, each of which has its own power engineering implication. Four types of capacity constraints are provided in equation (3) for the given upper bounds $\theta_{l m}^{\max }=\theta_{m l}^{\max }, P_{l m}^{\max }=P_{m l}^{\max }, S_{l m}^{\max }=S_{m l}^{\max }$ and $\Delta V_{l m}^{\max }=\Delta V_{m l}^{\max }$, where $\theta_{l m}$ denotes the angle difference $\measuredangle V_{l}-\measuredangle V_{m}$. Note that the constraint (3d) is equivalent to the line current limitation constraint in the context of this work, because each line has been modeled as a simple admittance and therefore $V_{l}-V_{m}$ is proportional to the line current. Henceforth, we assume that $\theta_{l m}^{\max }$ is less than $90^{\circ}$ due to the current practice in power networks. This can be assured by adding the constraint $\operatorname{Re}\left\{W_{l m}\right\}>0$ to the SDP relaxation, if necessary.

The capacity constraints given in (3) can all be cast as convex inequalities in $\mathbf{W}$, leading to the reformulated constraints in (4). To understand how the reformulation from $\mathbf{V}$ to $\mathbf{W}$ is carried out, consider the constraint (3a). This constraint is equivalent to $\left|\measuredangle\left(V_{l} V_{m}^{*}\right)\right| \leq \theta_{l m}^{\max }$ or

$$
\left|\frac{\operatorname{Im}\left\{V_{l} V_{m}^{*}\right\}}{\operatorname{Re}\left\{V_{l} V_{m}^{*}\right\}}\right| \leq \tan \left(\theta_{l m}^{\max }\right)
$$

Since $\theta_{l m}^{\max }$ is less than $90^{\circ}$ by assumption, the above inequality can be rewritten as

$$
\left|\operatorname{Im}\left\{V_{l} V_{m}^{*}\right\}\right| \leq \operatorname{Re}\left\{V_{l} V_{m}^{*}\right\} \tan \left(\theta_{l m}^{\max }\right)
$$

The convex constraint (4a) is obtained from the above inequality by replacing $V_{l} V_{m}^{*}$ with $W_{l m}$ and dropping the absolute value operator from the left side. Note that the absolute value is not important because the two constraints $\left|\theta_{l m}\right|,\left|\theta_{m l}\right| \leq \theta_{l m}^{\max }$ are equivalent to $\theta_{l m} \leq \theta_{l m}^{\max }$ and $\theta_{m l} \leq \theta_{l m}^{\max }$ all together (recall that $\theta_{l m}^{\max }=\theta_{m l}^{\max }$ ).

Theorem 1. Let $\alpha \in[0, \pi / 2)$ denote an arbitrary angle. Suppose that all voltage magnitudes are fixed at the nominal value of 1 per unit. Then, the capacity constraints in (3) are all mathematically equivalent and interchangeable through the upper bounds:

$$
\begin{aligned}
\theta_{l m}^{\max }(\alpha) & \triangleq \alpha \\
P_{l m}^{\max }(\alpha) & \triangleq \operatorname{Re}\left\{\left(1-e^{\alpha i}\right) y_{l m}^{*}\right\} \\
S_{l m}^{\max }(\alpha) & \triangleq\left|\left(1-e^{\alpha i}\right) y_{l m}^{*}\right| \\
\Delta V_{l m}^{\max }(\alpha) & \triangleq \sqrt{2(1-\cos (\alpha)) .}
\end{aligned}
$$

Proof: The proof may be found in the appendix.

Under relatively tight voltage conditions, the four capacity constraints in (3) give rise to very similar feasible regions for $\left(V_{l}, V_{m}\right)$ if the above upper bounds are employed. Given a certain level of deviation from the nominal voltage magnitude, it is possible to improve the above upper limits of the constraints by incorporating the deviation into these limits via solving a small optimization. In addition, given an upper bound for any of the constraints in (3), it is possible to design the upper bounds for the remaining three constraints in such a way that they all imply the constraint with the given upper bound. Since the maximum voltage deviation is usually small and less than $10 \%$ in general, it can be inferred from the above arguments that four common types of capacity constraints with different power engineering implications can be converted to each other with a good accuracy from a mathematical standpoint. To shed light on this fact, Figure 1 depicts the feasible region of $V_{m}$ for each of the constraints 


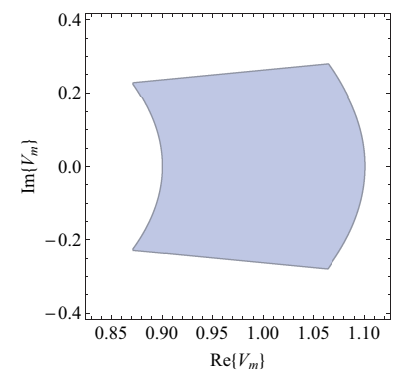

(a)

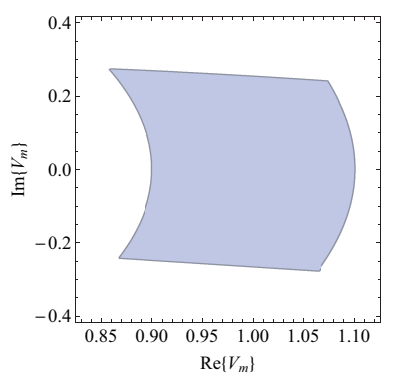

(b)

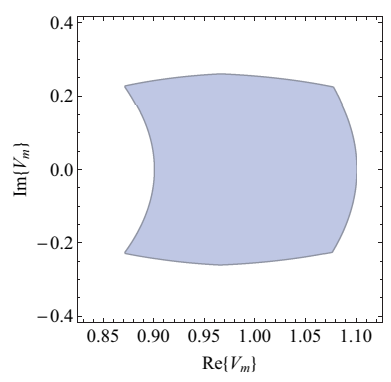

(c)

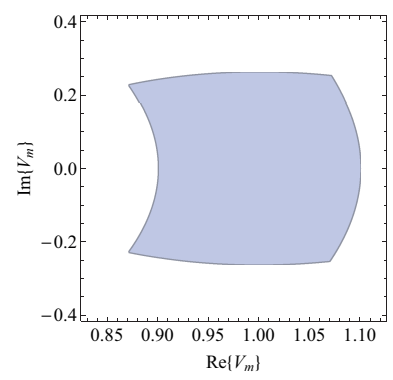

(d)

Fig. 1: Four feasible regions for voltage phasor $V_{m}$ (in p.u.) associated with the constraints in (3) in the case where $V_{l}$ is fixed at $1 \measuredangle 0^{\circ}$ (p.u.) and $0.9 \leq\left|V_{m}\right| \leq 1.1$ : (a) region for the line constraint (3a); (b) region for the line constraint (3b); (c) region for the line constraint (3c), and (d) region for the line constraint (3d).

in (3), where the upper bounds in (7) are deployed for the line $(l, m)$ under the following scenario: $\alpha=15^{\circ}$, the line admittance $y_{l m}=1 \measuredangle-80^{\circ}$ (p.u.), allowing a variable voltage magnitude for $V_{m}$ with the maximum permissible deviation of $10 \%$ from the nominal magnitude, and $V_{l}=1 \measuredangle 0^{\circ}$ (p.u.). It can be seen that the feasible regions are very similar and barely distinguishable from each other.

In the following subsection, we will show that this similarity (or equivalence in the extreme case of fixed voltage magnitudes) is no longer preserved after relaxation. In fact, it will be shown that the above capacity constraints behave very differently in the SDP relaxation (i.e., after removing the rank constraint $\operatorname{rank}\{\mathbf{W}\}=1$ ).

\section{SDP relaxation for a three-bus network}

It has been shown in [14] that the SDP relaxation is not exact for a specific three-bus power network with a triangular topology, provided one line has a very limited capacity. The capacity constraint in [14] has been formulated with respect to apparent power. It is imperative to study the interesting observation made in [14] because if the SDP relaxation cannot handle very simple cyclic networks, its application to mesh networks would be questionable. The result of [14] implies that the SDP relaxation is not necessarily exact for cyclic networks if the capacity constraint (3c) is employed. The high-level objective of this part is to make the surprising observation that the SDP relaxation becomes exact if the capacity constraint (3d) is used instead (this result will be proved later in the paper). To this end, we explore a scenario for which all four types of capacity constraints provided in (3) are equivalent but their convexified counterparts behave very differently. The goal is to show that the SDP relaxation is always exact only for one of these capacity constraints.

Consider the three-bus system depicted in Figure 2(a), which has been adopted from [14]. The parameters of this cyclic network are provided in Table I, where $z_{l m}=\frac{1}{y_{l m}}$ denotes the impedance of the line $(l, m)$. Assume that lines $(1,2)$ and $(2,3)$ have very high capacities, i.e.,

$$
\begin{aligned}
& \theta_{12}^{\max }=P_{12}^{\max }=S_{12}^{\max }=\Delta V_{12}^{\max }=\infty, \\
& \theta_{23}^{\max }=P_{23}^{\max }=S_{23}^{\max }=\Delta V_{23}^{\max }=\infty,
\end{aligned}
$$

while line $(1,3)$ has a very limited capacity. Since there are four ways to limit the flow over this line, we study four problems, each using only one of the capacity constraints given in (3) with its corresponding bound from (7). To this end, given an angle $\alpha$ belonging to the interval $\left[0,30^{\circ}\right]$, consider the following limits for these four problems:

$$
\begin{array}{ll}
\text { Problem A : } & \theta_{13} \leq \theta_{13}^{\max }(\alpha) \\
\text { Problem B : } & P_{13} \leq P_{13}^{\max }(\alpha) \\
\text { Problem C : } & S_{13} \leq S_{13}^{\max }(\alpha) \\
\text { Problem D : } & \Delta V_{13} \leq \Delta V_{13}^{\max }(\alpha)
\end{array}
$$

It is straightforward to verify that Problems A-D are equivalent due to the fact that they all lead to the same feasible set for the pair $\left(V_{1}, V_{3}\right)$. After removing the rank constraint from the OPF problem, these four problems become very distinct. To illustrate this property, we solve four relaxed SDP problems for the network depicted in Figure 2(a), corresponding to the equivalent Problems A-D. Figure 2(b) plots the optimal objective value of each of the four SDP relaxations as a function of $\alpha$ over the period $\alpha \in\left[0,30^{\circ}\right]$. Let $f^{\text {opt }}(\alpha)$ denote the solution of the original OPF problem. Each of the curves in Figure 2(b) is theoretically a lower bound on the function $f^{\text {opt }}(\alpha)$ in light of removing the non-convex constraint $\operatorname{rank}\{\mathbf{W}\}=1$. A few observations can be made here:

- The SDP relaxation for Problem D yields a rank-1 solution for all values of $\alpha$. Hence, the curve drawn in Figure 2(b) associated with Problem D represents the function $f^{\text {opt }}(\alpha)$, leading to the true solution of OPF.

- The curves for the SDP relaxations of Problems A-C do not overlap with $f^{\text {opt }}(\alpha)$ if $\alpha \in\left(0,7^{\circ}\right)$. Moreover, the gap between these curves and the function $f^{\text {opt }}(\alpha)$ is significant for certain values of $\alpha$.

- Figure 3 shows the case when a maximum of $10 \%$ offnominal voltage magnitude is allowed for each bus. In this case, Problem D is the only formulation that always results in a rank-1 solution.

In summary, three types of capacity constraints make the SDP relaxation inexact in general, while the last type of capacity constraint makes the SDP relaxation always exact. The current practice in power systems is to use Problem B 


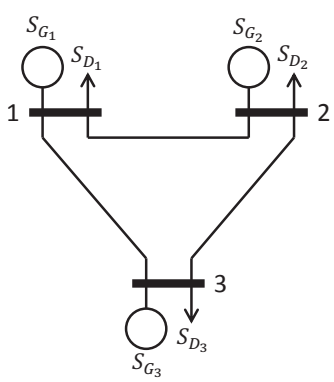

(a)

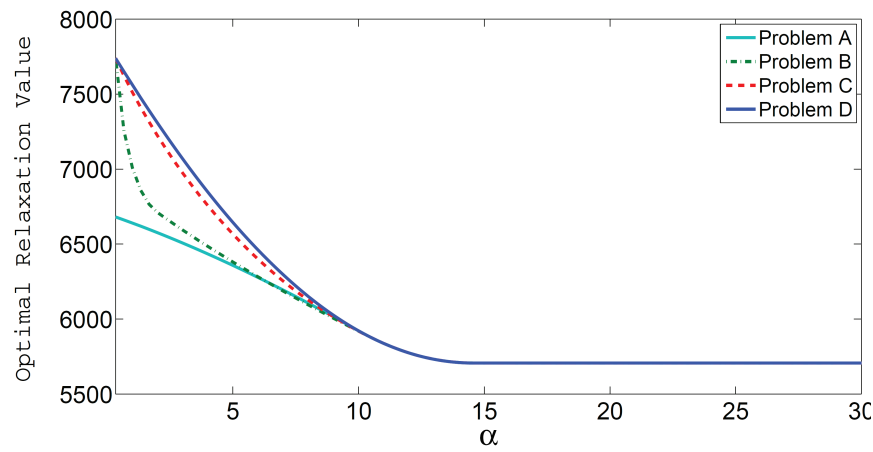

(b)

Fig. 2: (a) Three-bus system studied in Section II-C ; (b) optimal objective value of the SDP relaxation for Problems A-D.

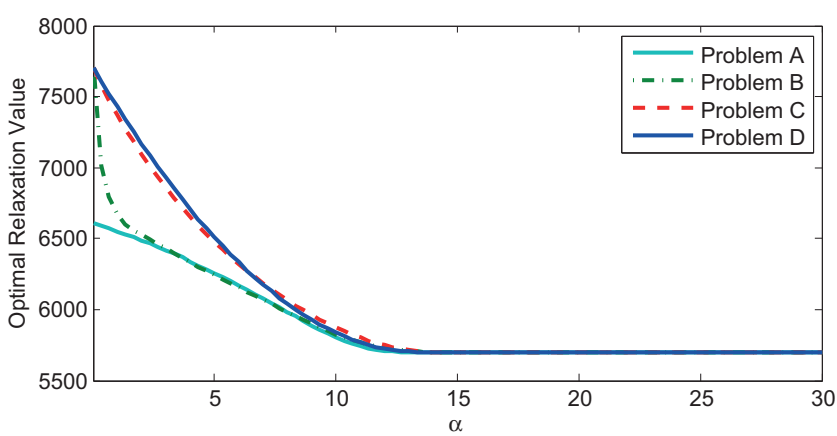

Fig. 3: Optimal objective value of the SDP relaxation for Problems A-D by allowing $10 \%$ off-nominal voltage magnitudes.

(due to its connection to DC OPF), but this example signifies that Problem D is the only one making the SDP relaxation a successful technique. Note that the capacity constraint considered in Problem D is closely related to the thermal loss, and therefore it may be natural to deploy Problem D for solving the OPF problem. Note also that if the OPF is defined in terms of multiple types of capacity constraints, the above reasoning justifies the need for converting the constraints into a single constraint of the form (3d).

Based on the methodology developed in [4] and [11], the above result can be interpreted in terms of the duality gap for OPF: there are four equivalent non-convex formulations of the OPF problem in the above example with the property that three of them have a nonzero duality gap in general while the last one always has a zero duality gap. This example reveals the fact that the problem formulation of OPF has a tremendous role in the success of the SDP relaxation, and in particular even equivalent formulations may become distinct after convexification. The observation made in this example will be proved for certain networks below.

Definition 1. A graph is called weakly cyclic if every edge of the graph belongs to at most one cycle in the graph.

Theorem 2. Consider the OPF problem (1) with the capacity constraint (3d) for a weakly-cyclic network with cycles of size 3. The following statements hold:

a) The SDP relaxation is exact in the lossless case, provided $Q_{k}^{\min }=-\infty$ for every $k \in \mathcal{N}$.

$$
\begin{aligned}
& f_{1}\left(P_{G_{1}}\right) \triangleq 0.11 P_{G_{1}}^{2}+5.0 P_{G_{1}} \\
& f_{2}\left(P_{G_{2}}\right) \triangleq 0.085 P_{G_{2}}^{2}+1.2 P_{G_{2}} \\
& f_{3}\left(P_{G_{3}}\right) \triangleq 0 \\
& z_{23}=0.025+0.750 \mathrm{i}, \quad S_{D_{1}}=110 \mathrm{MW} \\
& z_{31}=0.065+0.620 \mathrm{i}, \quad S_{D_{2}}=110 \mathrm{MW} \\
& z_{12}=0.042+0.900 \mathrm{i}, \quad S_{D_{3}}=95 \mathrm{MW} \\
& V_{k}^{\min }=V_{k}^{\max }=1 \quad \text { for } \quad k=1,2,3 \\
& \left(Q_{k}^{\min }, Q_{k}^{\max }\right)=(-\infty, \infty) \quad \text { for } \quad k=1,2,3 \\
& \left(P_{k}^{\min }, P_{k}^{\max }\right)=(-\infty, \infty) \quad \text { for } \quad k=1,2 \\
& P_{3}^{\min }=P_{3}^{\max }=0
\end{aligned}
$$

TABLE I: Parameters of the three-bus system drawn in Figure 2(a) with the base value 100 MVA.

b) The SDP relaxation is exact in the lossy case, provided $P_{k}^{\min }=Q_{k}^{\min }=-\infty$ and $Q_{k}^{\max }=+\infty$ for every $k \in \mathcal{N}$.

Proof: The proof may be found in the appendix.

Note that the statement of Theorem 2 cannot be generalized to the capacity constraints (3a)-(3c). This manifests the importance of the problem formulation and mathematical modeling.

\section{INJECTION REGION}

A power network under operation has a pair of flows $\left(P_{l m}, P_{m l}\right)$ over each line $(l, m) \in \mathcal{L}$ and a net injection $P_{k}$ at each bus $k \in \mathcal{N}$, where $P_{k}$ is indeed equal to $P_{G_{k}}-P_{D_{k}}$. This means that two vectors can be attributed to the network: (i) injection vector $\mathbf{P}=\left[\begin{array}{llll}P_{1} & P_{2} & \cdots & P_{n}\end{array}\right]$, (ii) flow vector $\mathbf{F}=$ $\left[P_{l m} \mid(l, m) \in \mathcal{L}\right]$. Due to the relation $P_{k}=\sum_{l \in \mathcal{N}(k)} P_{k l}$, there exists a matrix $M$ such that $\mathbf{P}=M \times \mathbf{F}$.

In order to understand the computational complexity of $\mathrm{OPF}$, it is beneficial to explore the feasible set for the injection vector. To this end, two notions of flow region and injection region will be defined in line with [19].

Definition 2. Define the flow region $\mathcal{F}$ as the set of all flow vectors $\mathbf{F}=\left[P_{l m} \mid(l, m) \in \mathcal{L}\right]$ for which there exists a voltage 


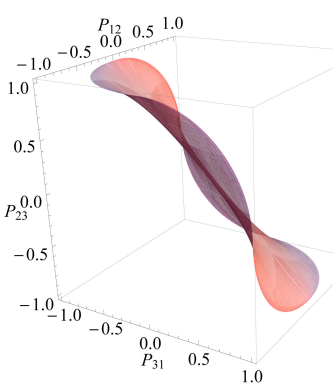

(a)

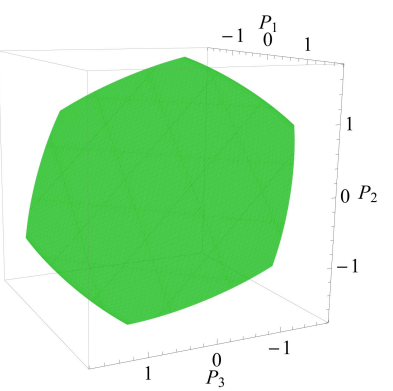

(b)
Fig. 4: (a) The reduced flow region $\mathcal{F}^{r}$ for a three-bus mesh network; (b) the injection region $\mathcal{P}$ for a three-bus mesh network.

phasors vector $\left[\begin{array}{llll}V_{1} & V_{2} & \cdots & V_{n}\end{array}\right]$ such that

$$
\begin{array}{lc}
P_{l m}=\operatorname{Re}\left\{V_{l}\left(V_{l}^{*}-V_{m}^{*}\right) y_{l m}^{*}\right\}, & (l, m) \in \mathcal{L} \\
\left|V_{l}-V_{m}\right| \leq \Delta V_{l m}^{\max }, & (l, m) \in \mathcal{L} \\
V_{k}^{\min } \leq\left|V_{k}\right| \leq V_{k}^{\max }, & k \in \mathcal{N}
\end{array}
$$

Define also the injection region $\mathcal{P}$ as $M \cdot \mathcal{F}$.

The above definition of the flow and injection regions captures the laws of physics, capacity constraints and voltage constraints. One can make this definition more comprehensive by incorporating reactive-power constraints.

Definition 3. Define the convexified flow region $\mathcal{F}_{c}$ as the set of all flow vectors $\mathbf{F}=\left[P_{l m} \mid(l, m) \in \mathcal{L}\right]$ for which there exists a matrix $\mathbf{W} \in \mathbb{H}_{+}^{n}$ such that

$$
\begin{aligned}
& P_{l m}=\operatorname{Re}\left\{\left(W_{l l}-W_{l m}\right) y_{l m}^{*}\right\} \\
& W_{l l}+W_{m m}-W_{l m}-W_{m l} \leq\left(\Delta V_{l m}^{\max }\right)^{2} \\
& \left(V_{k}^{\min }\right)^{2} \leq W_{k k} \leq\left(V_{k}^{\max }\right)^{2}
\end{aligned}
$$

for every $(l, m) \in \mathcal{L}$ and $k \in \mathcal{N}$. Define also the convexified injection region $\mathcal{P}_{c}$ as $M \cdot \mathcal{F}_{c}$.

It is straightforward to verify that $\mathcal{P} \subseteq \mathcal{P}_{c}$ and $\mathcal{F} \subseteq \mathcal{F}_{c}$.

\section{A. Lossless cycles}

A lossless network has the property that $P_{l m}+P_{m l}=0$ for every $(l, m) \in \mathcal{L}$, or alternatively $\operatorname{Re}\left\{y_{l m}\right\}=0$. Since realworld transmission networks are very close to being lossless, we study lossless mesh networks here. The flow region $\mathcal{F}$ has been defined in terms of two flows $P_{l m}$ and $P_{m l}$ for each line $(l, m) \in \mathcal{L}$. Due to the relation $P_{m l}=-P_{l m}$ for lossless networks, one can define a reduced flow region $\mathcal{F}^{r}$ based on one flow $P_{l m}$ for each line $(l, m)$.

The reduced flow region $\mathcal{F}^{r}$ has been plotted in Figure 4(a) for a cyclic three-bus network under the voltage setting $V_{k}^{\min }=V_{k}^{\max }$ for $k=1,2,3$ and some arbitrary capacity limits. This feasible set is a non-convex 2-dimensional curvy surface in $\mathbb{R}^{3}$. The corresponding injection region $\mathcal{P}$ can be obtained by applying an appropriate linear transformation to $\mathcal{F}^{r}$. Surprisingly, this set becomes convex, as depicted in Figure 4(b). More precisely, it can be shown that $\mathcal{P}=\mathcal{P}_{c}$ in this case. The goal of this part is to investigate the convexity of $\mathcal{P}$ for a single cycle. Assume for now that the power network is composed of a single cycle with the links $(1,2), \ldots,(n-1, n),(n, 1)$.

Theorem 3. Consider a lossless $n$-bus cycle with $n \geq 3$. The reduced flow region $\mathcal{F}^{r}$ is always non-convex if $V_{k}^{\min }=$ $V_{k}^{\max }, k=1,2, \ldots, n$.

Proof: The reduced flow region $\mathcal{F}^{r}$ consists of all vectors of the form $\left(\alpha_{1} \sin \left(\theta_{12}\right), \alpha_{2} \sin \left(\theta_{23}\right), \ldots \alpha_{n} \sin \left(\theta_{n 1}\right)\right)$, where $\theta_{12}+\theta_{23}+\cdots+\theta_{n 1}=0$ and $\alpha_{k}=\left|V_{k}\right|\left|V_{k+1}\right| \operatorname{Im}\left\{y_{k, k+1}^{*}\right\}$ for $k \in \mathcal{N}$. Therefore, $\mathcal{F}^{r}$ can be characterized in terms of $n-1$ independent angle differences $\theta_{12}, \ldots, \theta_{(n-1), n}$. This implies that $\mathcal{F}^{r}$ is an $(n-1)$-dimensional surface embedded in $\mathbb{R}^{n}$. On the other hand, this region cannot be embedded in $\mathbb{R}^{n-1}$ due to its non-zero curvature. Thus, $\mathcal{F}^{r}$ cannot be a convex subset of $\mathbb{R}^{n}$.

Since $V_{k}^{\min } \simeq V_{k}^{\max }$ in practice, it follows from Theorem 3 that the reduced flow region is expected to be non-convex under a normal operation.

Theorem 4. Consider a lossless n-bus cycle. The following statements hold:

a) For $n=2$ and $n=3$, the injection region $\mathcal{P}$ is convex and in particular $\mathcal{P}=\mathcal{P}_{c}$.

b) For $n \geq 5$, the injection region $\mathcal{P}$ is non-convex if

$$
\begin{aligned}
& V_{k}^{\min }=V_{k}^{\max }=V^{\max }, \quad k \in \mathcal{N} \\
& \Delta V_{l m}^{\max }=\Delta V^{\max }, \quad(l, m) \in \mathcal{L}
\end{aligned}
$$

for any arbitrary numbers $V^{\max }$ and $\Delta V^{\max }$.

Proof of Part (a): Consider an arbitrary injection vector $\bar{P}$ belonging to the convexified injection region $\mathcal{P}_{c}$. In order to prove Part (a), it suffices to show that $\bar{P}$ is contained in $\mathcal{P}$. Alternatively, it is enough to prove that the SDP relaxation of OPF with the capacity constraint (3d) and the parameters

$$
\begin{aligned}
& P_{k}^{\max }=P_{k}^{\min }=\bar{P}_{k} \\
& Q_{k}^{\max }=+\infty, \quad Q_{k}^{\min }=-\infty,
\end{aligned}
$$

has a rank-1 solution $\mathbf{W}$. This follows directly from Part (a) of Theorem 2.

Sketch of Proof for Part (b): Define

$$
\theta^{\max }=\cos ^{-1}\left(1-\frac{\left(\Delta V^{\max )^{2}}\right.}{2}\right)
$$

As pointed out in the proof of Theorem 3, the reduced flow region $\mathcal{F}^{r}$ contains all vectors of the form $\left(\alpha_{1} \sin \left(\theta_{12}\right), \alpha_{2} \sin \left(\theta_{23}\right), \ldots \alpha_{n} \sin \left(\theta_{n 1}\right)\right)$, where $\theta_{12}+\theta_{23}+$ $\ldots+\theta_{n 1}=0$ and $\left|\theta_{12}\right|, \ldots,\left|\theta_{n 1}\right| \leq \theta^{\max }$. Four observations can be made here:

i) The mapping from $\mathcal{F}^{r}$ to $\mathcal{P}$ is linear.

ii) The kernel of the map from $\mathcal{F}^{r}$ to $\mathcal{P}$ has dimension 1.

iii) Due to (i) and (ii), it can be proved that the restriction of $\mathcal{F}^{r}$ to the angles $\theta_{12}=\theta^{\max }$ and $\theta_{n 1}=-\theta^{\max }$ is a convex set whenever $\mathcal{P}$ is convex.

iv) The restriction of $\mathcal{F}^{r}$ to the angles $\theta_{12}=\theta^{\max }$ and $\theta_{n 1}=$ $-\theta^{\max }$ amounts to the reduced flow region for a single cycle of size $n-2$. In light of Theorem 3 , this set is nonconvex if $n-2 \geq 3$.

The proof of Part (b) follows from the above facts. 
Theorem 4 states that the injection region is convex only for small values of $n$.

\section{B. Weakly-cyclic networks}

In this part, the objective is to study the convexity of the injection region for a class of mesh networks. Although the class under investigation is simple and far from practical, its study gives rise to a good insight into the complexity of OPF. Notice that the injection region $\mathcal{P}$ is not necessarily convex for lossy networks. For example, the set $\mathcal{P}$ corresponding to a three-bus mesh network with nonzero loss is a curvy 2dimensional surface in $\mathbb{R}^{3}$. The objective of this part is to show that the front of this non-convex feasible set is convex in some sense.

Definition 4. Given a set $\mathcal{T} \subseteq \mathbb{R}^{n}$, define its Pareto front as the set of all points $\left(a_{1}, \ldots, a_{n}\right) \in \mathcal{T}$ for which there does not exist a different point $\left(b_{1}, \ldots, b_{n}\right)$ in $\mathcal{T}$ such that $b_{i} \leq a_{i}$ for $i=1, \ldots, n$.

Pareto front is an important subset of $\mathcal{T}$ because the solution of an arbitrary optimization over $\mathcal{T}$ with an increasing objective function must lie on the Pareto front of $\mathcal{T}$.

Theorem 5. The following statements hold for a weakly-cyclic network with cycles of size 3 :

a) If the network is lossless, then the injection region $\mathcal{P}$ is convex and in addition $\mathcal{P}=\mathcal{P}_{c}$.

b) If the network is lossy, then the injection region $\mathcal{P}$ and the convexified region $\mathcal{P}_{c}$ share the same Pareto front.

Proof: The proof of Part (a) of Theorem 4 also works for a general lossless weakly-cyclic network, leading to Part (a) of the present theorem.

In order to prove Part (b), we employ the same strategy as in the proof of Theorem 4. Assume that $\bar{P}$ belongs to the Pareto front of the convexified injection region $\mathcal{P}_{c}$. Consider the OPF problem (1) with the capacity constraint (3d) and let

$$
\begin{aligned}
P_{k}^{\max }=\bar{P}_{k}, & P_{k}^{\min }=-\infty, \\
Q_{k}^{\max }=+\infty, & Q_{k}^{\min }=-\infty .
\end{aligned}
$$

The objective function of the OPF problem can be replaced by a certain linear function in such a way that $\bar{P}$ becomes a solution of the SDP relaxation of this problem. On the other hand, it follows form Part (b) of Theorem 2 that there exists a solution $\left(\mathbf{P}^{\mathrm{opt}}, \mathbf{Q}^{\mathrm{opt}}, \mathbf{W}^{\mathrm{opt}}\right)$ for this problem where $\mathbf{W}^{\mathrm{opt}}$ is a rank-1 matrix. Since $\bar{P}$ belongs to the Pareto front of $\mathcal{P}_{c}$, we have $\mathbf{P}^{\text {opt }}=\bar{P}$. Hence, $\mathbf{P}^{\text {opt }}$ also belongs to $\mathcal{P}$ and that completes the proof.

\section{PENALIZED SDP RELAXATION}

So far, it has been shown that the SDP relaxation is exact for certain systems such as weakly-cyclic networks, provided a good mathematical formulation is deployed. Nevertheless, the SDP relaxation may not remain exact for mesh networks with large cycles. The objective of this section is to remedy this shortcoming for general networks. To this end, we first study the rank of the minimum-rank solution of the SDP relaxation and then introduce a penalization technique to enforce the rank of this solution matrix to become one. This will ultimately lead to a near-global solution of OPF with some measure of the optimality degree.

\section{A. Low-rank solution for SDP relaxation}

In this part, we first introduce some graph-theoretic parameters and then utilize them to relate the network topology to the existence of a low-rank solution for the SDP relaxation method.

Definition 5. Let $\mathcal{H}$ be a simple graph. Define $\mathcal{M}(\mathcal{H})$ as the set of all matrices $\mathbf{M} \in \mathbb{H}_{+}^{n}$ for which each off-diagonal entry $M_{l m}$ is nonzero if and only if $(l, m) \in \mathcal{E}_{\mathcal{H}}$. The minimum semidefinite rank (msr) of $\mathcal{H}$ is defined as [21]:

$$
\operatorname{msr}(\mathcal{H}) \triangleq \min \{\operatorname{rank}(\mathbf{M}) \mid \mathbf{M} \in \mathcal{M}(\mathcal{H})\} .
$$

The next theorem studies the rank of a solution of the SDP relaxation of the OPF problem under the load over satisfaction assumption

$$
P_{k}^{\min }=Q_{k}^{\min }=-\infty \quad \text { for } \quad k \in \mathcal{N} .
$$

A general version of this theorem with no extra assumption has been developed in the technical report [20].

Theorem 6. Consider the OPF problem given in (1) subject to the capacity constraints $(3 a),(3 b)$ and $(3 d)$, under the assumption $P_{k}^{\min }=Q_{k}^{\min }=-\infty$ for every $k \in \mathcal{N}$. If this problem is feasible, then its corresponding SDP relaxation has a solution $\left(\mathbf{W}^{\mathrm{opt}}, \mathbf{P}_{G}^{\mathrm{opt}}, \mathbf{Q}_{G}^{\mathrm{opt}}\right)$ such that

$$
\operatorname{rank}\left\{\mathbf{W}^{\text {opt }}\right\} \leq|\mathcal{H}|-\operatorname{msr}(\mathcal{H}),
$$

where $\mathcal{H}$ can be any arbitrary simple graph with the property that $\mathcal{V}_{\mathcal{H}}=\mathcal{N}$ and $\mathcal{L} \subseteq \mathcal{E}_{\mathcal{H}}$.

Proof: Since the OPF problem is feasible by assumption, there exists an optimal solution $\left(\mathbf{W}^{0}, \mathbf{P}_{G}^{\mathrm{opt}}, \mathbf{Q}_{G}^{\mathrm{opt}}\right)$ for this problem. Now, consider the optimization problem:

$$
\begin{array}{lll}
\min _{\mathbf{W} \in \mathbb{H}_{+}^{n}} & -\sum_{(l, m) \in \mathcal{E}_{\mathcal{H}}} \operatorname{Re}\left\{W_{l m}\right\} & \\
\text { s.t. } & W_{k k}=W_{k k}^{0}, & \\
& \operatorname{Re}\left\{W_{l m}\right\} \geq \mathcal{R}\left\{\left\{W_{l m}^{0}\right\},\right. & \\
& \operatorname{Im}\left\{W_{l m}\right\}=\operatorname{Im}\left\{W_{l m}^{0}\right\}, &
\end{array}
$$

Let $\mathbf{W}^{\text {opt }}$ denote an arbitrary solution of the above optimization. Since the resistance and inductance of each line $(l, m) \in \mathcal{L}$ are considered as nonnegative numbers in this paper, it is straightforward to verify that $\left(\mathbf{W}^{\mathrm{opt}}, \mathbf{P}_{G}^{\mathrm{opt}}, \mathbf{Q}_{G}^{\mathrm{opt}}\right)$ is an optimal solution of the SDP relaxation under the load over-satisfaction assumption. Now, it remains to prove that $\mathbf{W}^{\text {opt }}$ satisfies the inequality $\operatorname{rank}\left\{\mathbf{W}^{\text {opt }}\right\} \leq n-\operatorname{msr}(\mathcal{H})$.

To proceed with the proof, we aim to take the Lagrangian of Optimization (19). Let $\mathbf{A} \in \mathbb{H}_{+}^{n}$ denote the dual variable corresponding to the constraint $\mathbf{W} \succeq 0$. By noting that the positions of the nonzero off-diagonal entries of the matrix $\mathbf{A}$ correspond to the edges of the graph $\mathcal{H}$, it follows from the definition of "msr" that

$$
\operatorname{rank}\left\{\mathbf{A}^{\text {opt }}\right\} \geq \operatorname{msr}(\mathcal{H}) .
$$




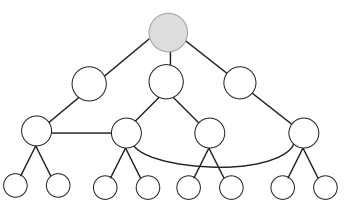

(a)

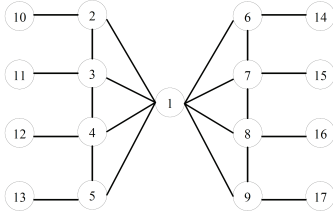

(b)
Fig. 5: Two graphs with $\eta=1$.

On the other hand, the complementary slackness condition trace $\left\{\mathbf{W}^{\text {opt }} \mathbf{A}^{\text {opt }}\right\}=0$ yields that

$$
\operatorname{rank}\left\{\mathbf{A}^{\mathrm{opt}}\right\}+\operatorname{rank}\left\{\mathbf{W}^{\mathrm{opt}}\right\} \leq n .
$$

The proof is completed by combining (20) and (21).

Roughly speaking, Theorem 6 aims to relate the computational complexity of the OPF problem to the topology of the power network by quantifying how inexact the SDP relaxation is.

Definition 6. Define $\eta(\mathcal{H})$ as the minimum number of vertices whose removal from the graph $\mathcal{H}$ eliminates all cycles of the graph.

To illustrate the definition of $\eta$, observe that this number is equal to 0 for a graph representing an acyclic network and is equal to 1 if all cycles of the network share a common node. Two graphs with $\eta=1$ are depicted in Figure 5.

Theorem 7. Consider the OPF problem given in (1) subject to the capacity constraints (3a), (3b) and (3d), under the assumption $P_{k}^{\min }=Q_{k}^{\min }=-\infty$ for every $k \in \mathcal{N}$. Let $\mathcal{H}$ be the graph that describes the topology of the power network under study. If the OPF problem is feasible, then its corresponding $S D P$ relaxation has a solution $\left(\mathbf{W}^{\mathrm{opt}}, \mathbf{P}_{G}^{\mathrm{opt}}, \mathbf{Q}_{G}^{\mathrm{opt}}\right)$ such that $\operatorname{rank}\left\{\mathbf{W}^{\text {opt }}\right\} \leq \eta(\mathcal{H})+1$.

Proof: Let $\mathcal{J}$ denote an induced subgraph of the power network with no cycles. One can expand $\mathcal{J}$ into a tree $\mathcal{T}$ by adding a minimal set of additional edges to this possibly disconnected subgraph. Let $\mathcal{H}^{\prime} \triangleq\left(\mathcal{V}_{\mathcal{H}}, \mathcal{E}_{\mathcal{H}} \cup \mathcal{E}_{\mathcal{T}}\right)$. According to Theorem, 6 there exists a solution $\left(\mathbf{W}^{\text {opt }}, \mathbf{P}_{G}^{\text {opt }}, \mathbf{Q}_{G}^{\text {opt }}\right)$ such that

$$
\operatorname{rank}\left\{\mathbf{W}^{\text {opt }}\right\} \leq\left|\mathcal{H}^{\prime}\right|-\operatorname{msr}\left(\mathcal{H}^{\prime}\right) .
$$

It also follows from [21] that

$$
\operatorname{msr}\left(\mathcal{H}^{\prime}\right) \geq\left|\mathcal{H}^{\prime}\right|-\eta\left(\mathcal{H}^{\prime}\right)-1 \text {. }
$$

Combining (22) and (23) yields

$$
\operatorname{rank}\left\{\mathbf{W}^{\mathrm{opt}}\right\} \leq \eta\left(\mathcal{H}^{\prime}\right)+1 \text {. }
$$

For an optimal choice of $\mathcal{J}$ with the maximum number of vertices $|\mathcal{J}|=|\mathcal{H}|-\eta(\mathcal{H})$, we have $\eta\left(\mathcal{H}^{\prime}\right)=\eta(\mathcal{H})$. This completes the proof.

There is a large body of literature on computing $\eta$, which signifies that this number is small for a very broad class of graphs, including mostly planar graphs. To illustrate the application of Theorem 7, consider the distribution network depicted in Figure 5(a). This network has three cycles, possibly used for exchanging renewable energy between the load buses without going through the feeder (the node shown in gray). Since removing this node eliminates all cycles of the network, it follows from Theorem 7 that the SDP relaxation of OPF has a solution with the property $\operatorname{rank}\left\{\mathbf{W}^{\text {opt }}\right\} \leq 2$.

Remark 1. The power balance equations (1a) and (1b) are equality constraints. One may relax these equations to inequality constraints so that each bus $k \in \mathcal{N}$ can be oversupplied. This notion is called over-satisfaction and has been considered in a number of papers (see [4], [2] and the references therein). The main idea is that whenever a power network operates under a normal condition, it is expected that the solution of the OPF problem remains intact or changes insignificantly under the load over-satisfaction assumption. The condition $P_{k}^{\min }=Q_{k}^{\min }=-\infty$ in Theorem 7 can be supplanted by the load over-satisfaction assumption

Remark 2. Given a general graph $\mathcal{H}$, finding the parameter $\eta(\mathcal{H})$ and its associated maximal induced forest $\mathcal{J}$ is known to be an NP-complete problem. Nevertheless, as shown in the proof of Theorem 7, any arbitrary set of nodes whose removal eliminates all cycles of the network leads to a solution $\mathbf{W}^{\text {opt }}$ together with an upper bound on its rank. In addition, the identification of $\mathcal{J}$ is mostly a one-time process and the algorithm proposed in [22] can be used for that purpose.

\section{B. Recovery of near-optimal solution for $O P F$}

As discussed in the preceding subsection, the SDP relaxation is expected to have a low-rank solution. This solution may be used to find an approximate rank-1 solution. Another technique is to enforce the SDP relaxation to eliminate the undesirable nonzero eigenvalues of the low-rank solution by incorporating a penalty term into its objective. The recent literature of compressed sensing suggests the penalty term $\varepsilon \times \operatorname{trace}\{\mathbf{W}\}$ for some coefficient $\varepsilon \in \mathbb{R}_{+}$[23]. However, this idea fails to work for OPF since all feasible solutions of the SDP relaxation have almost the same trace (because $V_{k}^{\mathrm{min}}$ and $V_{k}^{\max }$ are normally close to each other for $k=1, \ldots, n$ ). We propose a different penalty function in this paper.

Penalized SDP relaxation: This optimization is obtained from the SDP relaxation of the OPF problem by replacing its objective function with

$$
\sum_{k \in \mathcal{G}} f_{k}\left(P_{G_{k}}\right)+\varepsilon \sum_{k \in \mathcal{G}} Q_{G_{k}}
$$

for a given positive number $\varepsilon$.

There are two independent reasons behind the introduction of the penalty term $\sum_{k \in \mathcal{G}} Q_{G_{k}}$ :

- Consider a positive semidefinite matrix $\mathbf{X}$ with constant (fixed) diagonal entries $X_{11}, \ldots, X_{n n}$ and variable offdiagonal entries. If we maximize a weighted sum of the off-diagonal entries of $X$ with positive weights, then it turns out that $X_{l m}=\sqrt{X_{l l} X_{m m}}$ for all $l, m \in$ $\{1, \ldots, n\}$, in which case $\mathbf{X}$ becomes rank-1. Motivated by this fact, we employ the idea of elevating the offdiagonal entries of $\mathbf{W}$ to obtain a low-rank solution. For a lossless network, the above penalty term increases the 
weighted sum of the real parts of the off-diagonal entries of $\mathbf{W}$.

- Denote the set of all feasible vectors $\left(\mathbf{P}_{G}, \mathbf{Q}_{G}\right)$ satisfying the constraints of OPF as $\mathcal{T}$. The OPF problem minimizes the cost function $\sum_{k \in \mathcal{G}} f_{k}\left(P_{G_{k}}\right)$ over the projection of $\mathcal{T}$ onto the space for $\mathbf{P}_{G}$, which is referred to as $\mathcal{P}$ in this work. The projection from $\mathcal{T}$ to $\mathcal{P}$ maps multiple (possibly an uncountable number of) points into the same vector $\mathbf{P}_{G}$. This becomes a critical issue after removing the constraint $\operatorname{rank}\{\mathbf{W}\}=1$ from OPF. The main reason is that those multiple points with the same projection could correspond to different values of $\mathbf{W}$ with disparate ranks. The penalty term $\varepsilon \sum_{k \in \mathcal{G}} Q_{G_{k}}$ aims to guide the numerical algorithm by speculating that the right point $\left(\mathbf{P}_{G}, \mathbf{Q}_{G}\right)$ would cause the lowest reactive loss.

Let $\left(\mathbf{W}^{\text {opt }}, \mathbf{P}_{G}^{\text {opt }}, \mathbf{Q}_{G}^{\text {opt }}\right)$ and $\left(\mathbf{W}^{\varepsilon}, \mathbf{P}_{G}^{\varepsilon}, \mathbf{Q}_{G}^{\varepsilon}\right)$ denote arbitrary solutions of the SDP and penalized SDP relaxations, respectively. Assume that $\mathbf{W}^{\text {opt }}$ does not have rank 1, whereas $\mathbf{W}^{\varepsilon}$ has rank 1. It can be observed that the optimal objective value of OPF is lower and upper bounded by the respective numbers $\sum_{k \in \mathcal{G}} f_{k}\left(P_{G_{k}}^{\text {opt }}\right)$ and $\sum_{k \in \mathcal{G}} f_{k}\left(P_{G_{k}}^{\varepsilon}\right)$. Moreover, $\left(\mathbf{W}^{\varepsilon}, \mathbf{P}_{G}^{\varepsilon}, \mathbf{Q}_{G}^{\varepsilon}\right)$ can be mapped into the feasible solution $\left(\mathbf{V}^{\varepsilon}, \mathbf{P}_{G}^{\varepsilon}, \mathbf{Q}_{G}^{\varepsilon}\right)$ of the OPF problem, where $\mathbf{V}^{\varepsilon}\left(\mathbf{V}^{\varepsilon}\right)^{*}=\mathbf{W}^{\varepsilon}$. As a result, whenever the penalized SDP relaxation has a rank-1 solution, a feasible solution of OPF can be readily constructed and its sub-optimality degree can be measured subsequently. Note that a gradient descent algorithm can then be exploited to produce a local (if not global) solution from $\left(\mathbf{V}^{\varepsilon}, \mathbf{P}_{G}^{\varepsilon}, \mathbf{Q}_{G}^{\varepsilon}\right)$. Since the SDP relaxation of OPF possesses a low-rank solution in most cases, it is anticipated that the penalized SDP relaxation generates a global or near-global solution. We conducted extensive simulations on IEEE systems with more than 7000 different cost functions and observed that the penalized SDP relaxation always had a rank-1 solution. In addition, the obtained feasible solution of OPF was not only near optimal but also almost a local solution (satisfying the first order optimality conditions with some small error) in more than $95 \%$ of the trials. This observation will be elaborated in the next section. In what follows, we will provide partial theoretical results supporting our penalization technique.

Theorem 8. Consider a weakly-cyclic network with cycles of size 3. Given an arbitrary strictly positive number $\varepsilon$, every solution of the penalized SDP relaxation with the capacity constraint (4d) has rank-1, provided

a) $Q_{k}^{\min }=-\infty$ for every $k \in \mathcal{N}$ in the lossless case;

b) $P_{k}^{\min }=Q_{k}^{\min }=-\infty$ and $Q_{k}^{\max }=\infty$ for every $k \in \mathcal{N}$ in the lossy case.

Proof: This theorem can be proved in line with the technique developed in the proof of Theorem 2.

\section{Simulations}

Consider the IEEE 14-bus system with the cost function $\sum_{k \in \mathcal{G}} c_{k} P_{G_{k}}$, where the coefficients $c_{k}$ 's are provided in Table II(a). Let $\lambda_{1}$ and $\lambda_{2}$ denote the two largest eigenvalues of the matrix solution $\mathbf{W}_{\varepsilon}^{\text {opt }}$ of the penalized SDP relaxation. Solving this relaxation with $\varepsilon=0$ gives rise to $\lambda_{1}=15.1617$ and $\lambda_{2}=0.0138$, implying that the matrix $\mathbf{W}_{\varepsilon}^{\text {opt }}$ is nearly rank-1. However, $\lambda_{2}$ being nonzero is an impediment to the recovery of a feasible solution of OPF. To address this issue, we solve the penalized SDP relaxation with $\varepsilon=0.012$. This leads to a rank-1 matrix $\mathbf{W}_{\varepsilon}^{\text {opt }}$. The results are summarized in Table II(a). It can be seen that changing the penalty coefficient $\varepsilon$ from 0 to 0.012 has a negligible effect on $\mathbf{P}_{G}$ but a significant impact on $\mathbf{Q}_{G}$. As a result, the proposed penalization method corrects the vector of reactive powers and the upshot of this correction is the recovery of a feasible solution for OPF. Notice that the cost for this feasible solution is equal to 316.13 , while the optimal cost for the globally optimal solution of OPF is lower bounded by 316.08 , i.e., the solution of the SDP relaxation. This means that although it is hard to argue whether the feasible solution retrieved from the rank-1 matrix $\mathbf{W}_{\varepsilon}^{\text {opt }}$ for $\varepsilon=0.012$ is globally optimal for OPF, its sub-optimality degree is at least \%99.98 (this number is obtained by contrasting the cost 316.13 with the lower bound 316.08). It is even more interesting to note that the feasible solution recovered for OPF coincides with the solution found by the interior point method implemented in MATPOWER. This implies that the attained feasible solution is a local nearglobal (if not global) solution of OPF.

To gain some insight into the selection of the penalty coefficient $\varepsilon$, the cost $f_{\varepsilon}^{\text {opt }}=\sum_{k \in \mathcal{G}} f_{k}\left(P_{G_{k}}^{\varepsilon}\right)$ is plotted in Figure 6(a). It can be observed that this function is strictly increasing at the beginning, but there is a breakpoint at which the function becomes almost flat. Interestingly, the matrix $\mathbf{W}_{\varepsilon}^{\text {opt }}$ has rank 2 before the breakpoint $\varepsilon=0.012$ and rank 1 after this point. Consequently, there is a range of values for $\varepsilon$ (as opposed to a single number) that makes the matrix $\mathbf{W}_{\varepsilon}^{\text {opt }}$ rank 1 and keeps the cost at the lowest level (due to the almost flat part of the curve $f_{\varepsilon}^{\text {opt }}$ ).

The above experiment was repeated on two very extreme cases for IEEE 30 and 57-bus systems with linear cost functions. The results are summarized in Tables II(b)-(c) and Figures 6(b)-(c). The observations made for each of these cases conform with the previous ones: (i) there is a turning point at which the cost function $f_{\varepsilon}^{\text {opt }}$ becomes almost flat and concurrently the matrix $\mathbf{W}_{\varepsilon}^{\text {opt }}$ becomes rank 1 , (ii) the feasible solution of OPF recovered from a rank-1 matrix $\mathbf{W}_{\varepsilon}^{\text {opt }}$ is not only near-optimal but also a local solution. The phenomenon of the "almost flat part segment" in the curve $f_{\varepsilon}^{\text {opt }}$ has been observed in numerous cases examined by the authors for which the (unpenalized) SDP relaxation did not have a rank-1 solution.

Some modifications on the IEEE test cases and other well known examples have been proposed in [15] and [16], which make the SDP relaxation method fail to work. Consider the case "modified 14-bus" from [16] and "modified 118-bus" from [15] to evaluate the performance of the penalized SDP method:

- For the case "modified 14-bus" from [16], the (unpenalized) SDP lower bound on the optimal cost of the solution is 8092.36. A rank-1 solution can be obtained at $\varepsilon=80$ with the cost 8092.72 .

- For the case "modified 118-bus" from [15], the diagram of the optimal cost versus the penalty coefficient $\varepsilon$ is shown in Figure 7. This system has at least 3 local 


\begin{tabular}{|c|c|c|c|c|c|}
\hline \multicolumn{5}{|c|}{ IEEE-14 } \\
\hline \hline \multicolumn{2}{|c|}{$\boldsymbol{\epsilon}$} & \multicolumn{2}{|c|}{0} & \multicolumn{2}{c|}{0.012} \\
\hline $\boldsymbol{\lambda}_{\mathbf{1}}$ & \multicolumn{2}{|c|}{15.1617} & \multicolumn{2}{c|}{15.1340} \\
\hline $\boldsymbol{\lambda}_{\mathbf{2}}$ & \multicolumn{2}{|c|}{0.0138} & \multicolumn{2}{c|}{0} \\
\hline \multicolumn{2}{|c|}{ Cost } & \multicolumn{2}{|c|}{$\$ 316.08$} & \multicolumn{2}{c|}{$\$ 316.13$} \\
\hline \hline $\boldsymbol{k}$ & $\boldsymbol{c}_{\boldsymbol{k}}$ & $\boldsymbol{P}_{\boldsymbol{G}_{\boldsymbol{k}}}$ & $\boldsymbol{Q}_{\boldsymbol{G}_{\boldsymbol{k}}}$ & $\boldsymbol{P}_{\boldsymbol{G}_{\boldsymbol{k}}}$ & $\boldsymbol{Q}_{\boldsymbol{G}_{\boldsymbol{k}}}$ \\
\hline 1 & 3 & 25.36 & 0 & 25.38 & 0.85 \\
\hline 2 & 1 & 140 & 25.44 & 140 & 22.25 \\
\hline 3 & 4 & 0 & 28.77 & 0 & 27.11 \\
\hline 6 & 1 & 100 & -6 & 100 & -6 \\
\hline 8 & 4 & 0 & 9.16 & 0 & 6.42 \\
\hline
\end{tabular}

(a)

\begin{tabular}{|c|c|c|c|c|c|}
\hline \multicolumn{5}{|c|}{ IEEE-30 } \\
\hline \hline \multicolumn{2}{|c|}{$\boldsymbol{\epsilon}$} & \multicolumn{2}{c|}{0} & \multicolumn{2}{c|}{0.55} \\
\hline \multicolumn{2}{|c|}{$\boldsymbol{\lambda}_{\mathbf{1}}$} & 30.6789 & \multicolumn{2}{c|}{30.8677} \\
\hline \multicolumn{2}{|c|}{$\boldsymbol{\lambda}_{\boldsymbol{2}}$} & \multicolumn{2}{c|}{0.4986} & \multicolumn{2}{c|}{0} \\
\hline \multicolumn{2}{|c|}{ Cost } & \multicolumn{2}{c|}{$\$ 414.34$} & \multicolumn{2}{c|}{$\$ 438.40$} \\
\hline \hline $\boldsymbol{k}$ & $\boldsymbol{c}_{\boldsymbol{k}}$ & $\boldsymbol{P}_{\boldsymbol{G}_{\boldsymbol{k}}}$ & $\boldsymbol{Q}_{\boldsymbol{G}_{\boldsymbol{k}}}$ & $\boldsymbol{P}_{\boldsymbol{G}_{\boldsymbol{k}}}$ & $\boldsymbol{Q}_{\boldsymbol{G}_{\boldsymbol{k}}}$ \\
\hline 1 & 1 & 80 & 11.11 & 80 & -4.60 \\
\hline 2 & 10 & 0 & 39.16 & 0 & -2.10 \\
\hline 13 & 1 & 40 & 44.70 & 40 & 44.70 \\
\hline 33 & 10 & 23.98 & 35.26 & 27.32 & 33.36 \\
\hline 23 & 100 & 0 & 33.39 & 0 & 15.62 \\
\hline 27 & 1 & 54.55 & 25.65 & 45.22 & 21.33 \\
\hline
\end{tabular}

(b)

\begin{tabular}{|c|c|c|c|c|c|}
\hline \multicolumn{5}{|c|}{ IEEE-57 } \\
\hline \hline \multicolumn{2}{|c|}{$\boldsymbol{\epsilon}$} & \multicolumn{2}{c|}{0} & \multicolumn{2}{c|}{1.5} \\
\hline \multicolumn{2}{|c|}{$\boldsymbol{\lambda}_{\mathbf{1}}$} & \multicolumn{2}{c|}{57.1776} & \multicolumn{2}{c|}{56.8887} \\
\hline \multicolumn{2}{|c|}{$\boldsymbol{\lambda}_{\mathbf{2}}$} & \multicolumn{2}{c|}{0.0767} & \multicolumn{2}{c|}{0} \\
\hline \multicolumn{2}{|c|}{ Cost } & \multicolumn{2}{c|}{$\$ 259.70$} & \multicolumn{2}{c|}{$\$ 272.73$} \\
\hline \hline $\boldsymbol{k}$ & $\boldsymbol{c}_{\boldsymbol{k}}$ & $\boldsymbol{P}_{\boldsymbol{G}_{\boldsymbol{k}}}$ & $\boldsymbol{Q}_{\boldsymbol{G}_{\boldsymbol{k}}}$ & $\boldsymbol{P}_{\boldsymbol{G}_{\boldsymbol{k}}}$ & $\boldsymbol{Q}_{\boldsymbol{G}_{\boldsymbol{k}}}$ \\
\hline 1 & 0.1 & 575.88 & 78.60 & 575.88 & 111.87 \\
\hline 2 & 0.1 & 100 & 50 & 100 & 50 \\
\hline 3 & 100 & 0 & 60 & 0 & 44.29 \\
\hline 6 & 0.1 & 100 & 25 & 100 & 25 \\
\hline 8 & 10 & 13.11 & 117.90 & 14.41 & 159.64 \\
\hline 9 & 0.1 & 100 & 9 & 100 & 9 \\
\hline 12 & 0.1 & 410 & 96.91 & 410 & -6.29 \\
\hline
\end{tabular}

(c)

TABLE II: Three case studies for IEEE systems: (a) IEEE-14; (b) IEEE-30; (c) IEEE-57.

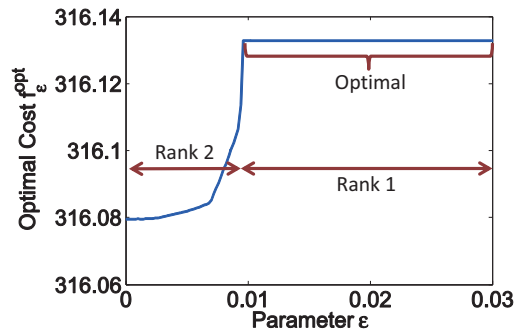

(a)

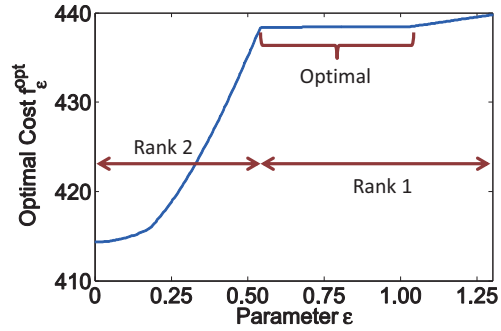

(b)

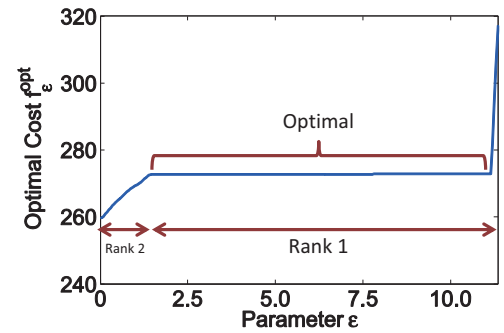

(c)

Fig. 6: (a) IEEE-14; (b) IEEE-30; (c) IEEE-57

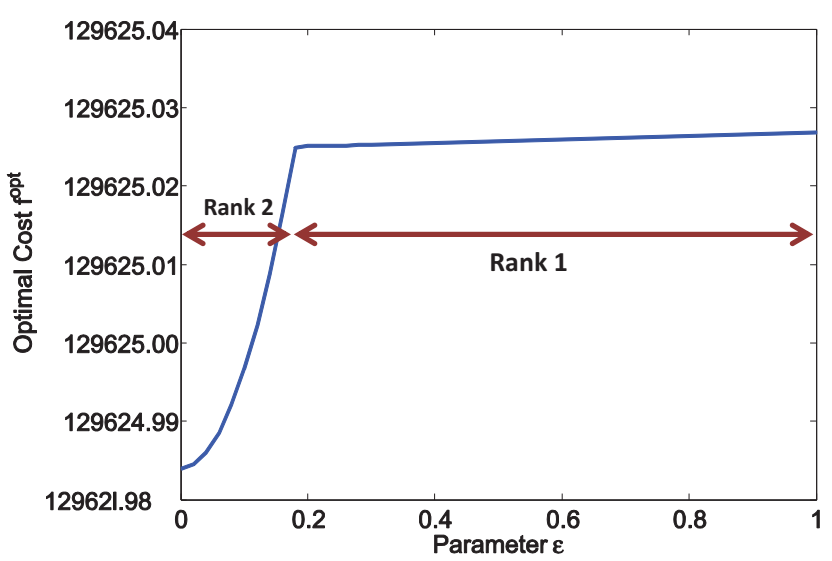

Fig. 7: The modified 118-bus system [15]

minima with the associated costs $129625.03,177984.32$ and 195695.54. The penalized SDP relaxation gives rise to the best minimum among these local minima for $\varepsilon \simeq 0.2$.

To demonstrate the merit of the penalized SDP relaxation, we generated more than 7000 cost functions for IEEE 14, 30 and 57-bus systems with the network parameters obtained from MATPOWER test data files-including constraints limiting the apparent power for each line-where the cost coefficients $c_{k}$ 's were chosen from the discrete set $\{1,2,3,4\}$. We then conducted the above experiment on all these generated
OPF problems and tabulated the findings in the supplement http://www.columbia.edu/ rm3122/research.html. The results are encapsulated below:

- There were many cases for which the penalized SDP relaxation with $\varepsilon=0$ had a rank-1 solution. This means that the unpenalized SDP relaxation was able to find a global solution of OPF in many cases.

- There were cases for which the numerical solution of the SDP relaxation was not rank 1, but the penalized SDP relaxation produced a rank-1 solution for a very small number $\varepsilon$. For example, this occurs for the IEEE30 bus system with $c_{k}=1$ for which $\mathbf{W}^{\text {opt }}$ has two non-zero eigenvalues 32.3437 and 0.0112 , while $\mathbf{W}_{\epsilon}^{\mathrm{opt}}$ has only one nonzero eigenvalue equal to 32.3433 for $\epsilon=10^{-5}$. Under this circumstance, the SDP relaxation has multiple solutions, including a hidden rank-1 solution that can be obtained through the penalized SDP relaxation with a very small $\epsilon$.

- In many cases, there exists an $\varepsilon_{1}>0$ such that the penalized SDP relaxation always yields a rank-1 solution for every $\varepsilon>\varepsilon_{1}$ and that there exists an interval $\left(\varepsilon_{1}, \varepsilon_{2}\right)$ in which the resulting cost changes very slightly (as shown in Figures 6 and 7). Although the cost can increase dramatically for $\varepsilon>\varepsilon_{2}$, like the case shown in Figure 6(c), we observed that the interval $\left(\varepsilon_{1}, \varepsilon_{2}\right)$ of interest is relatively large and an $\varepsilon$ inside that interval can be spotted with 2 or 3 trial and errors.

- Whenever the SDP relaxation failed to work for each 
of the generated cases (counting over 7000 OPFs), the penalized SDP relaxation always had a rank-1 solution with a carefully chosen $\varepsilon$. In addition, the recovered near-optimal solution of OPF almost satisfied the KKT conditions (subject to some small error) in $100 \%, 96.6 \%$ and $95.8 \%$ of cases for IEEE 14, 30 and 57-bus systems, respectively. This means that these sub-optimal points would be almost globally optimal.

\section{CONCLUSiOns}

We have recently shown that the semidefinite programming (SDP) can be used to find a global solution of the OPF problem for IEEE benchmark power systems. Although the exactness of the SDP relaxation for acyclic networks has been successfully proved, a recent work has witnessed the failure of this technique for a three-bus cyclic network. Inspired by this observation, the present paper is concerned with understanding the limitations of the SDP relaxation for cyclic power networks. First, it is shown that the injection region of a weaklycyclic network with cycles of size 3 is convex in the lossless case and has a convex Pareto front in the lossy case. It is then proved that the SDP relaxation works for this type of network. This result implies that the failure of the SDP relaxation for a three-bus network recently reported in the literature can be fixed by utilizing a good modeling of the line capacity. As a more general result, it is then shown that whenever the SDP relaxation does not work, it is expected to have a low-rank solution in practice. Finally, a penalized SDP relaxation is proposed from which a near-global solution of OPF may be recovered. The performance of this method is tested on IEEE systems with over 7000 different cost functions.

\section{REFERENCES}

[1] J. A. Momoh, M. E. El-Hawary, and R. Adapa, "A review of selected optimal power flow literature to 1993. part i: Nonlinear and quadratic programming approaches," IEEE Transactions on Power Systems, 1999.

[2] R. Baldick, Applied Optimization: Formulation and Algorithms for Engineering Systems. Cambridge, 2006.

[3] K. S. Pandya and S. K. Joshi, "A survey of optimal power flow methods," Journal of Theoretical and Applied Information Technology, 2008.

[4] J. Lavaei and S. H. Low, "Zero duality gap in optimal power flow problem," IEEE Transactions on Power Systems, vol. 27, no. 1, pp. 92-107, 2012.

[5] A. Y. S. Lam, B. Zhang, A. Dominguez-Garcia, and D. Tse, "Optimal distributed voltage regulation in power distribution networks," Submitted for publication, 2012.

[6] Y. Weng, Q. Li, R. Negi, and M. Ilic, "Semidefinite programming for power system state estimation," IEEE Power \& Energy Society General Meeting, 2012.

[7] D. K. Molzahn, B. C. Lesieutre, and C. L. DeMarco, "A sufcient condition for power flow insolvability with applications to voltage stability margins," http://arxiv.org/pdf/1204.6285.pdf, 2012.

[8] E. Dall' Anese, H. Zhu, and G. Giannakis, "Distributed optimal power flow for smart microgrids," to appear in IEEE Transactions on Smart Grid, 2013.

[9] S. Sojoudi and S. Low, "Optimal charging of plug-in hybrid electric vehicles in smart grids," IEEE Power and Energy Society General Meeting, 2011.

[10] S. Ghosh, D. A. Iancu, D. Katz-Rogozhnikov, D. T. Phan, and M. S. Squillante, "Power generation management under time-varying power and demand conditions," IEEE Power \& Energy Society General Meeting, 2011.

[11] S. Sojoudi and J. Lavaei, "Physics of power networks makes hard optimization problems easy to solve," IEEE Power \& Energy Society General Meeting, 2012.
[12] B. Zhang and D. Tse, "Geometry of feasible injection region of power networks," 49th Annual Allerton Conference, 2011.

[13] S. Bose, D. F. Gayme, S. Low, and M. K. Chandy, "Optimal power flow over tree networks," Proceedings of the Forth-Ninth Annual Allerton Conference, pp. 1342-1348, 2011.

[14] B. Lesieutre, D. Molzahn, A. Borden, and C. L. DeMarco, "Examining the limits of the application of semidefinite programming to power flow problems," 49th Annual Allerton Conference, 2011.

[15] W. Bukhsh, A. Grothey, K. McKinnon, and P. Trodden, "Local solutions of the optimal power flow problem," Power Systems, IEEE Transactions on, vol. 28, no. 4, pp. 4780-4788, 2013.

[16] A. Gopalakrishnan, A. U. Raghunathan, D. Nikovski, and L. T. Biegler, "Global optimization of optimal power flow using a branch \& bound algorithm," in Communication, Control, and Computing (Allerton), 2012 50th Annual Allerton Conference on. IEEE, 2012, pp. 609-616.

[17] D. T. Phan, "Lagrangian duality and branch-and-bound algorithms for optimal power flow," Operations Research, vol. 60, no. 2, pp. 275-285, 2012.

[18] J. Lavaei, "Zero duality gap for classical OPF problem convexifies fundamental nonlinear power problems," American Control Conference, 2011.

[19] J. Lavaei, B. Zhang, and D. Tse, "Geometry of power flows in tree networks," IEEE Power \& Energy Society General Meeting, 2012.

[20] R. Madani, G. Fazelnia, S. Sojoudi, and J. Lavaei, "Finding low-rank solutions of sparse linear matrix inequalities using convex optimization," Technical Report, 2014. [Online]. Available: http://www.ee.columbia.edu/ lavaei/LMI_Low_Rank.pdf

[21] M. Booth, P. Hackney, B. Harris, C. R. Johnson, M. Lay, L. H. Mitchel, S. Narayan, A. Pascoe, K.Steinmetz, B. Sutton, and W. Wang, "On the minimum rank among positive semidefinite matrices with a given graph," SIAM J. on Matrix Analysis and Applications, vol. 30, p. 731740, 2008.

[22] I. Razgon, "Exact computation of maximum induced forest," in Algorithm Theory-SWAT 2006. Springer, 2006, pp. 160-171.

[23] B. Recht, M. Fazel, and P. A. Parrilo, "Guaranteed minimum rank solutions to linear matrix equations via nuclear norm minimization," SIAM Review, vol. 52, pp. 471-501, 2010.

\section{APPENDIX}

Proof of Theorem 1: In order to prove the equivalence of the constraints (3a) and (3b) at the nominal voltage magnitudes, notice that

$$
\begin{aligned}
P_{l m} & =\operatorname{Re}\left\{V_{l}\left(V_{l}^{*}-V_{m}^{*}\right) y_{l m}^{*}\right\} \\
& =\operatorname{Re}\left\{\left(1-e^{\theta_{l m} \mathrm{i}}\right) y_{l m}^{*}\right\} \\
& =\left|y_{l m}^{*}\right|\left[\cos \left(\measuredangle y_{l m}^{*}\right)-\cos \left(\theta_{l m}+\measuredangle y_{l m}^{*}\right)\right] .
\end{aligned}
$$

By inspecting the sinusoidal term inside the expression of $P_{l m}$, it is straightforward to verify that $\left|P_{l m}\right|$ attains its maximum value at $\theta_{l m}=\alpha$. For the constraints (3c) and (3d), one can write:

$$
\begin{aligned}
\left|S_{l m}\right|^{2} & =\left|V_{l}\left(V_{l}^{*}-V_{m}^{*}\right) y_{l m}^{*}\right|^{2} \\
& =\left|y_{l m}^{*}\right|^{2}\left|\left(1-e^{\mathrm{i} \theta_{l m}}\right)\right|^{2} \\
& =2\left|y_{l m}^{*}\right|^{2}\left(1-\cos \left(\theta_{l m}\right)\right)
\end{aligned}
$$

and

$$
\begin{aligned}
\left|V_{l}-V_{m}\right|^{2} & =\left|V_{l}\right|^{2}+\left|V_{m}\right|^{2}-2\left|V_{l}\right|\left|V_{m}\right| \cos \left(\theta_{l m}\right) \\
& =2\left(1-\cos \left(\theta_{l m}\right)\right) .
\end{aligned}
$$

By inspecting the term $\cos \left(\theta_{l m}\right)$ and using the assumption $\alpha \in[0, \pi / 2)$, it follows from the above relations that

$$
\begin{aligned}
\theta_{l m} \in[-\alpha, \alpha] & \Leftrightarrow\left|S_{l m}\right| \leq S_{l m}^{\max }(\alpha) \\
& \Leftrightarrow\left|V_{l}-V_{m}\right| \leq \Delta V_{l m}^{\max }(\alpha)
\end{aligned}
$$

This completes the proof. 
Proof of Theorem 2: The proof is trivial for a 2-bus network. Assume for now that the network is composed of a single cycle of size 3 . In order to prove the theorem in this case, consider an arbitrary solution $\left(\mathbf{P}_{G}^{\text {init }}, \mathbf{Q}_{G}^{\text {init }}, \mathbf{W}^{\text {init }}\right)$ of the SDP relaxation. It suffices to show that there exists another solution $\left(\mathbf{P}_{G}^{\text {opt }}, \mathbf{Q}_{G}^{\text {opt }}, \mathbf{W}^{\text {opt }}\right)$ with the same cost as $\left(\mathbf{P}_{G}^{\text {init }}, \mathbf{Q}_{G}^{\text {init }}, \mathbf{W}^{\text {init }}\right)$ such that $\operatorname{rank}\left\{\mathbf{W}^{\text {opt }}\right\}=1$. Alternatively, it is enough to prove that the feasibility problem

$$
\begin{aligned}
& P_{k}^{\min } \leq P_{D_{k}}+\sum_{l \in \mathcal{N}(k)} \operatorname{Re}\left\{\left(W_{k k}-W_{k l}\right) y_{k l}^{*}\right\} \leq P_{G_{k}}^{\text {init }} \\
& Q_{k}^{\min } \leq Q_{D_{k}}+\sum_{l \in \mathcal{N}(k)} \operatorname{Im}\left\{\left(W_{k k}-W_{k l}\right) y_{k l}^{*}\right\} \leq Q_{G_{k}}^{\max } \\
& \left(V_{k}^{\min }\right)^{2} \leq W_{k k} \leq\left(V_{k}^{\max }\right)^{2} \\
& W_{l l}+W_{m m}-W_{l m}-W_{m l} \leq\left(\Delta V_{l m}^{\max }\right)^{2} \\
& \mathbf{W} \succeq 0
\end{aligned}
$$

$\forall k \in \mathcal{N},(l, m) \in \mathcal{L}$, has a rank-1 solution $\mathbf{W}^{\text {opt }}$. To this end, we convert the above feasibility problem into an optimization by adding the objective function

$$
\min _{\mathbf{W} \in \mathbb{H}^{n}}-\sum_{k \in \mathcal{G}} Q_{G_{k}}
$$

to the problem. Let $\underline{\nu}_{k}, \underline{\lambda}_{k}, \underline{\mu}_{k} \in \mathbb{R}_{+}, \bar{\nu}_{k}, \bar{\lambda}_{k}, \bar{\mu}_{k}, \psi_{l m} \in \mathbb{R}_{+}$, and $\mathbf{A} \in \mathbb{H}_{+}^{3}$ denote the Lagrange multipliers corresponding to the lower bounding constraints (31a), (31b), (31c), upper bounding constraints (31a), (31b), (31c), (31d), and (31e), respectively. It can be shown that

$$
\begin{aligned}
A_{l m}= & -\operatorname{Im}\left\{y_{l m}^{*}\right\}-\psi_{l m}-\psi_{m l} \\
& -\frac{\left(\bar{\nu}_{l}-\underline{\nu}_{l}\right) y_{l m}^{*}+\left(\bar{\nu}_{m}-\underline{\nu}_{m}\right) y_{l m}}{2} \\
& -\frac{\left(\bar{\lambda}_{l}-\underline{\lambda}_{l}\right) y_{l m}^{*}-\left(\bar{\lambda}_{m}-\underline{\lambda}_{m}\right) y_{l m}}{2 \mathbf{i}}
\end{aligned}
$$

for every $(l, m) \in \mathcal{L}$. Define $\nu_{k} \triangleq \bar{\nu}_{k}-\underline{\nu}_{k}$ and $\lambda_{k} \triangleq \bar{\lambda}_{k}-\underline{\lambda}_{k}$, for every $k \in \mathcal{N}$. Then, (33) can be rewritten as

$$
\begin{aligned}
A_{l m}= & -\psi_{l m}-\psi_{m l} \\
& -\operatorname{Re}\left\{y_{l m}^{*}\right\}\left[\frac{\nu_{l}+\nu_{m}-\left(\lambda_{l}-\lambda_{m}\right) \mathbf{i}}{2}\right] \\
& -\operatorname{Im}\left\{y_{l m}^{*}\right\}\left[1+\frac{\lambda_{l}+\lambda_{m}+\left(\nu_{l}-\nu_{m}\right) \mathbf{i}}{2}\right]
\end{aligned}
$$

for every $(l, m) \in \mathcal{L}$. Moreover, the complementary slackness condition yields that trace $\left\{\mathbf{W}^{\text {opt }} \mathbf{A}^{\text {opt }}\right\}=0$ at optimality. To prove that $\mathbf{W}^{\text {opt }}$ has rank 1 , it suffices to show that $\mathbf{A}^{\text {opt }}$ has rank $n-1=2$. To prove the later statement by contradiction, assume that $\mathbf{A}^{\text {opt }}$ has rank 1. Therefore, the determinant of each $2 \times 2$ submatrix of $\mathbf{A}^{\text {opt }}$ must be zero. In particular,

$$
\begin{gathered}
\operatorname{det}\left[\begin{array}{cc}
A_{12}^{\mathrm{opt}} & A_{13}^{\mathrm{opt}} \\
A_{22}^{\mathrm{opt}} & A_{23}^{\mathrm{opt}}
\end{array}\right]=A_{12}^{\mathrm{opt}} A_{23}^{\mathrm{opt}}-A_{13}^{\mathrm{opt}} A_{22}^{\mathrm{opt}}=0 \\
\Longrightarrow \quad \measuredangle A_{12}^{\mathrm{opt}}+\measuredangle A_{23}^{\mathrm{opt}}-\measuredangle A_{13}^{\mathrm{opt}}=\measuredangle A_{22}^{\mathrm{opt}} .
\end{gathered}
$$

Since $\mathbf{A}^{\mathrm{opt}}$ is Hermitian, we have

$$
\measuredangle A_{22}^{\mathrm{opt}}=0 \quad \text { and } \quad \measuredangle A_{13}^{\mathrm{opt}}=-\measuredangle A_{31}^{\mathrm{opt}}
$$

and hence the following relation must hold:

$$
\measuredangle A_{12}^{\mathrm{opt}}+\measuredangle A_{23}^{\mathrm{opt}}+\measuredangle A_{31}^{\mathrm{opt}}=0 .
$$

On the other hand, under the assumptions of the theorem, we have

$$
\operatorname{Re}\left\{y_{l m}^{*}\right\}=0, \quad \lambda_{k} \geq 0
$$

for Part (a) and

$$
\lambda_{k}=0, \quad \nu_{k} \geq 0
$$

for Part (b). Hence, it can be concluded from (34) and each set of equations (39) or (40) that

$$
\begin{array}{r}
\operatorname{Re}\left\{A_{12}^{\text {opt }}\right\}, \operatorname{Re}\left\{A_{23}^{\text {opt }}\right\}, \operatorname{Re}\left\{A_{31}^{\text {opt }}\right\}<0 \\
\frac{\operatorname{Im}\left\{A_{12}^{\text {opt }}\right\}}{\operatorname{Im}\left\{y_{12}^{*}\right\}}+\frac{\operatorname{Im}\left\{A_{23}^{\text {opt }}\right\}}{\operatorname{Im}\left\{y_{23}^{*}\right\}}+\frac{\operatorname{Im}\left\{A_{31}^{\text {opt }}\right\}}{\operatorname{Im}\left\{y_{31}^{*}\right\}}=0 .
\end{array}
$$

(recall that $y_{l m}^{*}$ has nonnegative real and imaginary parts due to the positivity assumption of the resistance and reactance of each line). It can be concluded from (41b) that the elements of the set $\left\{\operatorname{Im}\left\{A_{12}^{\mathrm{opt}}\right\}, \operatorname{Im}\left\{A_{23}^{\mathrm{opt}}\right\}, \operatorname{Im}\left\{A_{31}^{\mathrm{opt}}\right\}\right\}$ are neither all positive nor all negative. With no loss of generality, it suffice to study the following two cases:

i) If

$$
\operatorname{Im}\left\{A_{12}^{\mathrm{opt}}\right\}, \operatorname{Im}\left\{A_{23}^{\mathrm{opt}}\right\} \geq 0 \text { and } \operatorname{Im}\left\{A_{31}^{\mathrm{opt}}\right\} \leq 0,
$$

then according to (41a), we have:

$$
\begin{aligned}
\pi / 2 & <\measuredangle A_{12}^{\mathrm{opt}} \leq \pi \\
\pi / 2 & <\measuredangle A_{23}^{\mathrm{opt}} \leq \pi \\
\pi & \leq \measuredangle A_{31}^{\mathrm{opt}}<3 \pi / 2 .
\end{aligned}
$$

ii) If

$$
\operatorname{Im}\left\{A_{12}^{\text {opt }}\right\}, \operatorname{Im}\left\{A_{23}^{\text {opt }}\right\} \leq 0 \quad \text { and } \quad \operatorname{Im}\left\{A_{31}^{\text {opt }}\right\} \geq 0,
$$

then according to (41a), we have:

$$
\begin{aligned}
\pi & \leq \measuredangle A_{12}^{\mathrm{opt}}<3 \pi / 2 \\
\pi & \leq \measuredangle A_{23}^{\mathrm{opt}}<3 \pi / 2 \\
\pi / 2 & <\measuredangle A_{31}^{\mathrm{opt}} \leq \pi
\end{aligned}
$$

Both (43) and (45) yield that

$$
2 \pi<\measuredangle A_{12}^{\mathrm{opt}}+\measuredangle A_{23}^{\mathrm{opt}}+\measuredangle A_{31}^{\mathrm{opt}}<4 \pi
$$

implying that the angle relation (38) does not hold. This contradiction completes the proof for both Parts (a) and (b).

For a general network with multiple cycles, let $\mathcal{O}$ denote the set of all 3-bus cyclic subgraphs of the power network. Define $\overline{\mathcal{O}}$ as the set of all bridge edges (i.e., those edges whose removal makes the graph disconnected). By adapting the proof delineated above for a single link and a single cycle, it can be shown that the SDP relaxation has a solution $\mathbf{W}^{\text {opt }}$ with the property

$$
\operatorname{rank}\left(W^{\text {opt }}(\mathcal{S})\right)=1 \text { for all } \mathcal{S} \in \mathcal{O} \cup \overline{\mathcal{O}}
$$


where $W^{\text {opt }}(\mathcal{S})$ is a sub-matrix of $\mathbf{W}^{\text {opt }}$ obtained by picking every row and column of $\mathbf{W}^{\text {opt }}$ whose index corresponds to a vertex of the subgraph $\mathcal{S}$. The above relation yields that

$$
\left|W^{\mathrm{opt}}\right|=\sqrt{W_{l l}^{\mathrm{opt}} W_{m m}^{\mathrm{opt}}}, \quad(l, m) \in \mathcal{L}
$$

and that

$$
\measuredangle W^{\mathrm{opt}}(\mathcal{S})_{1,2}+\measuredangle W^{\mathrm{opt}}(\mathcal{S})_{2,3}+\measuredangle W^{\mathrm{opt}}(\mathcal{S})_{3,1}=0
$$

for every $\mathcal{S} \in \mathcal{O}$. It follows from the above equation that there exist some angles $\theta_{1}, \ldots, \theta_{n} \in[-\pi, \pi]$ such that

$$
\theta_{l}-\theta_{m}=\measuredangle W_{l m}^{\mathrm{opt}} \text { for all }(l, m) \in \mathcal{L}
$$

Now, it is easy to verity that $\mathbf{V}^{\text {opt }}\left(\mathbf{V}^{\text {opt }}\right)^{*}$ is a rank-1 solution of the SDP relaxation, where

$$
\mathbf{V}^{\mathrm{opt}}=\left[\sqrt{W_{11}^{\mathrm{opt}}} e^{-\theta_{1} \mathrm{i}}, \sqrt{W_{22}^{\mathrm{opt}}} e^{-\theta_{2} \mathrm{i}}, \ldots, \sqrt{W_{n n}^{\mathrm{opt}}} e^{-\theta_{n} \mathrm{i}}\right]^{*}
$$

This completes the proof.

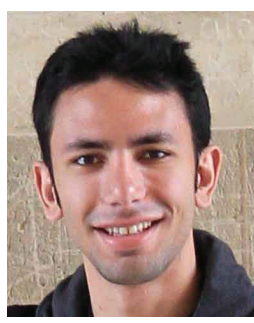

Ramtin Madani received the B.Sc. and M.Sc. degrees in electrical engineering from Sharif University of Technology, Tehran, Iran, in 2010 and 2012 respectively. He is currently working towards the $\mathrm{Ph} . \mathrm{D}$. degree in the Department of Electrical Engineering, Columbia University, New York, NY, USA. His current research interests include optimization over graphs, optimal power networks \& smart grids, distributed control and nonlinear optimization. $\mathrm{He}$ has worked in many areas of optimization theory, communications, and signal processing.

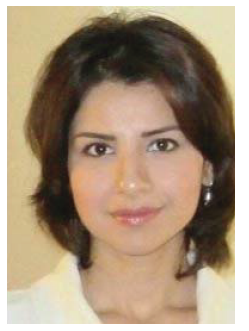

Somayeh Sojoudi received the M.A.Sc. degree in electrical engineering from Concordia University, Montral, QC, Canada, in 2008, and the Ph.D. degree in control and dynamical systems from the California Institute of Technology (Caltech), Pasadena, CA, USA, in 2013. She is a Postdoctoral Scholar in NYU comprehensive epilepsy center, New York, NY, USA. Her research interests include neuroscience, power systems, and communication networks. Dr. Sojoudi has received a postgraduate scholarship from the Natural Sciences and Engineering Research Council of Canada (NSERC). She is also a recipient of the $2008 \mathrm{~F}$. A Gerard Prize (Best Master's Thesis Award) of the Faculty of Engineering and Computer Science, Concordia University.

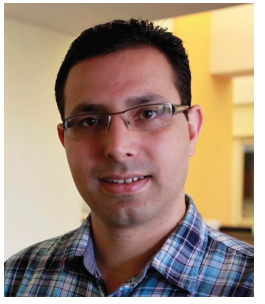

Javad Lavaei is an Assistant Professor in the Department of Electrical Engineering at Columbia University, New York, NY, USA. He obtained his $\mathrm{Ph} . \mathrm{D}$. degree in Control \& Dynamical Systems from California Institute of Technology, Pasadena, California, USA, and held a one-year postdoc position jointly with Electrical Engineering and Precourt Institute for Energy at Stanford University, Palo Alto, California, USA. He is recipient of the Milton and Francis Clauser Doctoral Prize for the best university-wide $\mathrm{Ph} . \mathrm{D}$. thesis. His research interests include power systems, networking, distributed computation, optimization, and control theory. Javad Lavaei is a senior member of IEEE and has won several awards, including Office of Naval Research Young Investigator Award, NSF CAREER Award, Google Faculty Research Award, Resonate Award, the Canadian Governor Generals Gold Medal, Northeastern Association of Graduate Schools Masters Thesis Award, New Face of Engineering in 2011, and Silver Medal in the 1999 International Mathematical Olympiad. 